\title{
Nicotine-Induced Upregulation of Native Neuronal Nicotinic Receptors Is Caused by Multiple Mechanisms
}

\author{
Anitha P. Govind, Heather Walsh, and William N. Green \\ Department of Neurobiology, University of Chicago, Chicago, Illinois 60637
}

\begin{abstract}
Nicotine causes changes in brain nicotinic acetylcholine receptors (nAChRs) during smoking that initiate addiction. Nicotine-induced upregulation is the long-lasting increase in $\mathrm{nAChR}$ radioligand binding sites in brain resulting from exposure. The mechanisms causing upregulation are not established. Many different mechanisms have been reported with the assumption that there is a single underlying cause. Using live rat cortical neurons, we examined for the first time how exposure and withdrawal of nicotine shape the kinetics of native $\alpha 4 \beta 2$-containing $\mathrm{nAChR}$ upregulation in real time. Upregulation kinetics demonstrates that at least two different mechanisms underlie this phenomenon. First, a transient upregulation occurs that rapidly reverses, faster than $n A C h R$ degradation, and corresponds to $n A C h R$ conformational changes as assayed by conformational-dependent, subunit-specific antibodies. Second, a long-lasting process occurs correlating with increases in $\mathrm{nAChR}$ numbers caused by decreased proteasomal subunit degradation. Previous radioligand binding measurements to brain tissue have measured the second process and largely missed the first. We conclude that nicotine-induced upregulation is composed of multiple processes occurring at different rates with different underlying causes.
\end{abstract}

\section{Introduction}

Nicotine is the major addiction-causing component in tobacco. Addiction is initiated by nicotine binding to high-affinity sites on nicotinic acetylcholine receptors ( $\mathrm{nAChRs)}$ in brain. $\mathrm{nAChRs}$ are membrane proteins belonging to the cys-loop family of neurotransmitter-gated ion channels (Karlin and Akabas, 1995; Albuquerque et al., 2009). Neuronal nAChRs are comprised of eight different $\alpha$ subunits: $\alpha 2-\alpha 7 ; \alpha 9-\alpha 10$; and three other $\beta$ subunits, $\beta 2-\beta 4$. Different nAChR subtypes are pentamers composed of two or more different $\alpha$ and $\beta$ subunits, with the exception of the $\alpha$-bungarotoxin-binding subtype, which only contains $\alpha 7$ subunits (Drisdel and Green, 2000), resulting in distinct pharmacology and function in vivo (Sargent, 1993; McGehee and Role, 1995). The majority of high-affinity nicotinebinding sites in brain contain $\alpha 4$ and $\beta 2$ subunits (Whiting and Lindstrom, 1988). In addition, there are other less characterized subtypes including receptors composed of $\alpha 4, \beta 2$, and $\alpha 5$ subunits (Conroy and Berg, 1998); $\alpha 3, \beta 2$, and $\beta 4$ subunits (Xu et al., 1999; Parker et al., 2004); and $\alpha 6$ with other subunits (Klink et al., 2001; Cui et al., 2003; Parker et al., 2004).

Nicotine-induced upregulation is linked to different processes in nicotine addiction, including sensitization (Vezina et al., 2007;

\footnotetext{
Received Oct. 27, 2011; revised Dec. 9, 2011; accepted Jan. 4, 2012.

Author contributions: A.P.G., H.W., and W.N.G. designed research; A.P.G. and H.W. performed research; A.P.G., H.W., and W.N.G. analyzed data; A.P.G. and W.N.G. wrote the paper.

This work was supported by National Institutes of Health Grants NS043782 and DA019695, the Peter F. McManus Foundation (W.N.G.), and fellowships from the Marine Biological Laboratory in Woods Hole, MA. We thank Dr. Scott Rogers for generously providing some of the Abs used in this study. We also thank Dr. Okunola Jeyifous for helping with primary neuronal cultures of cortex and Dr. Sarah Antinone for critical reading and comments about this paper. The authors declare no competing financial interests.

Correspondence should be addressed to Dr. William N. Green, Department of Neurobiology, University of Chicago, 947 E. 58th Street, Chicago, IL 60637. E-mail:wgreen@midway.uchicago.edu.

DOI:10.1523/JNEUROSCI.5438-11.2012

Copyright $\odot 2012$ the authors $\quad 0270-6474 / 12 / 322227-12 \$ 15.00 / 0$
}

Govind et al., 2009) and withdrawal (De Biasi and Dani, 2011). Upregulation occurs when nicotine exposure increases highaffinity nicotine-binding sites in brain, measured by radiolabeled agonists such as nicotine (Marks et al., 1983; Schwartz and Kellar, 1983; Benwell et al., 1988; Breese et al., 1997) or epibatidine (Perry et al., 1999). Several factors limit studies examining the mechanisms underlying upregulation. First, the number of brain nAChR subtypes is not known because of their low levels. Another limitation is that radioligand binding to brain nAChRs is performed on membrane preparations or autoradiography sections, and events that occur in live neurons may be missed. An alternative approach has been heterologous expression of different subunit combinations in mammalian cell lines or Xenopus oocytes (Peng et al., 1994; Hsu et al., 1996; Fenster et al., 1999). The disadvantage of heterologous expression is that nAChR subunit composition, while defined, may not correspond to that of native nAChRs. Non-neuronal cells may also lack brain-specific factors that regulate upregulation in brain.

Here, we assayed nicotine-induced upregulation of native nAChRs using live cortical neurons, which allowed real-time measurements of nAChR upregulation. We examined the initiation of upregulation and its reversal with nicotine withdrawal. The kinetics of native $\mathrm{nAChR}$ upregulation is biphasic revealing different processes that cause nicotine-induced upregulation. The first process is rapid and did not correlate with changes in the number of nAChRs, but did correlate with conformationaldependent binding of antibodies (Abs). The data are consistent with $\mathrm{nAChR}$ conformational changes causing transitions between a resting low-affinity state and an upregulated high-affinity state, as previously proposed (Vallejo et al., 2005). The second process is much slower and correlated with slowed $\beta 2$ subunit endoplasmic reticulum (ER) degradation, which causes increased subunit assembly and increased insertion of cell-surface 
nAChRs (Darsow et al., 2005). Nicotine-induced upregulation is, therefore, caused by multiple processes, contrary to the assumption that upregulation results from a single underlying cause.

\section{Materials and Methods}

Abs used. nAChR subunit-specific antibody mAb299 ( $\alpha 4)$ was purchased from Covance; mAb270 ( $\beta 2$ ) from Developmental Studies Hybridoma Bank at the University of Iowa; anti- $\beta 2$ SC $(C-20)$ from Santa Cruz Biotechnology; $6963(\alpha 4), 4860(\alpha 3)$, and $4886(\beta 4)$ were kind gifts from Professor Scott Rogers (University of Utah, Salt Lake City, UT); anti-HA (rabbit polyclonal) from Bethyl Laboratories; anti-actin from SigmaAldrich; and anti-glutamate decarboxylase (GAD) 65 from Millipore. Anti-glial fibrillary acidic protein (GFAP) and anti-microtubuleassociated protein 2 (MAP-2) were both purchased from Cell Signaling Technology. Secondary antibody anti-rabbit HRP was obtained from Chappel; anti-goat HRP from Santa Cruz Biotechnology; and Alexa Fluor anti-rat 488/568, Alexa Fluor anti-rabbit 647, and Alexa Fluor anti-Mouse 568 from Molecular Probes.

Cell culture. HEK 293 cells (tSA201) stably expressing rat $\alpha 4 \beta 2$ $\mathrm{nAChRs}$ were generated in our laboratory, and the $\beta 2$ subunit expresses an HA epitope at the $\mathrm{C}$ terminus as previously described by Vallejo et al. (2005). Cells were maintained in DMEM supplemented with 10,000 $\mathrm{U} / \mathrm{ml}$ penicillin and streptomycin (Invitrogen), 10\% calf serum (HyClone), and Hygromycin B (EMD Chemicals) at $37^{\circ} \mathrm{C}$ in the presence of $5 \% \mathrm{CO}_{2}$.

Primary cultures of rat cortical neurons. Cortical neurons were cultured using the protocol listed under the Johns Hopkins Medicine web site (http://www.hopkinsmedicine.org/institute_basic_biomedical_sciences/ research_centers/metabolism_obesity_research/protocols/) with minor modifications. Cortex from Sprague Dawley rat pups of either sex at embryonic day 18 (E18) were dissected out and cut into small pieces. To dissociate the cells, the cortical pieces were incubated in $0.25 \%$ trypsin (Invitrogen) for $30 \mathrm{~min}$, followed by washing once with FBS (HyClone) and thrice with HBSS (Invitrogen). After the final wash, the tissue was triturated using fire-polished glass pipettes. The dissociated cells were passed through a $70 \mu \mathrm{m}$ cell strainer (BD Biosciences) to remove debris. The cells were centrifuged at $1500 \mathrm{rpm}$ for $5 \mathrm{~min}$ and resuspended in neurobasal medium supplemented with B27 (Invitrogen). The cells are plated in $6 \mathrm{~cm}$ tissue culture dishes precoated with poly-D-lysine (SigmaAldrich) with an average density of $2 \times 10^{6}$. Half of the media was replaced every 3-4 d until the experiments were finished.

Metabolic labeling, immunoprecipitation, and affinity purification. Newly synthesized subunits were labeled with ${ }^{35} \mathrm{~S}$-methionine and cysteine $(1175 \mathrm{Ci} / \mathrm{mmol}$; Easy tag EXPRESS S35 protein-labeling mix, PerkinElmer Life and Analytical Science). Cultures were starved in methionine-free DMEM for $15 \mathrm{~min}$ at $37^{\circ} \mathrm{C}$, incubated in $5 \% \mathrm{CO}_{2}$, and labeled in $1.5 \mathrm{ml}$ of methionine-free medium supplemented with 111 $\mu \mathrm{Ci}$ of the ${ }^{35} \mathrm{~S}$ mix for $30 \mathrm{~min}$. After labeling cells were washed thrice with ice-cold DMEM and maintained in DMEM at $37^{\circ} \mathrm{C}$ and incubated in $5 \%$ $\mathrm{CO}_{2}$ in the presence or absence of nicotine for varying time intervals. At each chase time point, cells were scraped off the plate in $1 \mathrm{ml}$ of PBS, centrifuged at $2400 \mathrm{rpm}$ for $3 \mathrm{~min}$, and resuspended in lysis buffer (150 mм NaCl, 5 mм EDTA, 50 mм Tris, pH 7.4, 0.02\% NaN3, plus $1 \%$ Triton $\mathrm{X}-100$ ) containing protease inhibitors [ $2 \mathrm{~mm}$ phenylmethylsulfonyl fluoride (PMSF), $2 \mathrm{~mm} N$-ethylmaleimide, and chymostatin, pepstatin, leupeptin, and tosyllysine chloromethyl ketone at $10 \mu \mathrm{g} / \mathrm{ml}]$. Insoluble material was pelleted by centrifugation, and the resultant ${ }^{35} \mathrm{~S}$-labeled lysates were incubated with anti-HA polyclonal antibody, $\operatorname{mAb} 270(\beta 2)$, AChR $\beta 2 \mathrm{C}-20$ $(\beta 2)$, mAb $299(\alpha 4)$, or $6963(\alpha 4)$ overnight at $4^{\circ} \mathrm{C}$. Antibody-antigen complexes were precipitated with protein G-Sepharose (GE Healthcare), and purified subunits were analyzed on a $4-8 \%$ gradient with SDS-PAGE. The gel was dried and exposed to Bio-Rad screen, and the auto-rad was developed using a Bio-Rad molecular imager pharos-FX (Bio-Rad). Quantification was done using ImageJ.

Nicotine-induced upregulation and ${ }^{125}$ I-epibatidine binding. HEK cells stably expressing $\alpha 4 \beta 2 \mathrm{HA}$ maintained at $37^{\circ} \mathrm{C}$ in DMEM were treated with $10 \mu \mathrm{M}$ nicotine for $17 \mathrm{~h}$. For intact counts, cells were washed three times with PBS and collected by gentle agitation with PBS followed by incubation with $5 \mathrm{~nm}{ }^{125} \mathrm{I}$-epibatadine ( ${ }^{125} \mathrm{I}$-epi) $(2200 \mathrm{Ci} / \mathrm{mmol}$; PerkinElmer Life and Analytical Science) for $20 \mathrm{~min}$. The cells were harvested onto filter paper GF/B filters presoaked in $0.5 \%$ polyethyleneimine and washed with PBS using a 24-channel cell harvester (Brandel). Radioactivity of bound ${ }^{125} \mathrm{I}$-epi was determined by gamma counting (PerkinElmer Life and Analytical Science). Nonspecific binding was estimated by ${ }^{125} \mathrm{I}$-epi binding done on parallel samples preincubated with 1 mm nicotine.

To obtain antibody-precipitated counts, the cells were solubilized in lysis buffer (see above) following $17 \mathrm{~h}$ of nicotine exposure. Insoluble material was pelleted by centrifugation, which removed $\sim 25 \%$ of the available ${ }^{125} \mathrm{I}$-epi binding sites. The lysates were subjected to immunoprecipitation with various subunit-specific Abs. The antigen-antibody complexes were pulled down using protein G-Sepharose beads (GE Healthcare). Following immunoprecipitation the beads were bound with $2.5 \mathrm{~nm}{ }^{125} \mathrm{I}$-epi for $1 \mathrm{~h}$. Binding was done in the presence of $1 \mathrm{~mm}$ nicotine to determine the nonspecific counts. The radioactivity associated with the beads was determined using gamma counting. The Abs used were tested on cell lines expressing $\alpha 4 \beta 4, \alpha 3 \beta 2$, or $\alpha 6 \beta 2$ to determine their specificity. The $\beta 2$-specific Abs did not precipitated measurable ${ }^{125} \mathrm{I}$-epi counts from $\alpha 4 \beta 4$-expressing cells, and $\alpha 4$-specific Abs did not precipitate ${ }^{125} \mathrm{I}$-epi counts from $\alpha 3 \beta 2$ - or $\alpha 6 \beta 2$-expressing cells.

Primary cultures of cortical neurons were treated with $1 \mu \mathrm{M}$ nicotine for $17 \mathrm{~h}$. The cells were gently scraped off the plates and resuspended in 1 $\mathrm{nM}{ }^{125} \mathrm{I}$-epi for $20 \mathrm{~min}$. The neurons were vacuum harvested to GF/B filter (Brandel), and the specific radioactivity associated with the filters was measured as described above. In the case of antibody precipitation, the neurons were exposed to nicotine as described above and lysed with lysis buffer containing 1\% Triton X-100 and protease inhibitors. The neuronal lysates were incubated with subunit-specific Abs overnight followed by protein G-Sepharose precipitation. The immunoprecipitated receptors were bound with $0.5 \mathrm{~nm}{ }^{125} \mathrm{I}$-epi for $1 \mathrm{~h}$. The beads were washed three times with lysis buffer, and the radioactivity remaining on the beads was determined using gamma counting.

RT-PCR analysis. Total RNA was isolated from primary cortical cultures using a Purelink RNA mini kit (Invitrogen). Total RNA was reverse transcribed using random hexamer primers and multiscribe reverse transcriptase according to manufacturer's protocol (RT kit; Applied Biosystem). Reactions lacking RNA or reverse transcriptase served as control for DNA contamination or genomic amplification, respectively. PCR primers were designed based on published protocols (Sheffield et al., 2000; Rogers et al., 2001 ) and the conditions used therein were followed. Primer pairs are listed for each nicotinic receptor subunit: nAChR $\beta 2$, sense 59-GGAGTGGGAA GATTACCGCCTCA, antisense 5'-AGTCGTCGTGGTTCTCGTTGCG; nAChR $\beta 3$, sense 5' -GCTGAACACGAAGACGC, antisense 5' -GCAAAGAC AGTCACC; nAChR $\beta 4$, sense 5'-AGAGTGCCTGCAAGATTGAG, antisense $5^{\prime}$-AGCTGACTGCAGACTTAGGA; nAChR $\alpha 2$, sense 5'-GATCTG GATCCCAGACATTG, antisense 5'-CGCCGATGAGTGGGATGACC; nAChR $\alpha 3$, sense $5^{\prime}$-GGTGGATGACAAGACCAAAGC, antisense 5' -AGGG CAGGTAGAAGACAAGCA; nAChR $\alpha 4$, sense 5'-CACGGTCTTCGTGCT CAATGT, antisense 5'-CCTTGGTTGCAGATGTCACTC; nAChR $\alpha 5$, sense 5'-GCTGCGCTGCTCTTGATGGT, antisense 5' -CGTATGTCCACG AGCCGAAT; nAChR $\alpha 6$, sense 5' -GCTTCATCCGGCCAGTGG, antisense 5'-GCAAAGAGTCACTTTCTCG; nAChR $\alpha 7$, sense 5' -CGCTGGTTCCCT TTTGATGTG, antisense $5^{\prime}$-CTATCAAGGGCACAGAATCAG; $\beta$-actin, sense $5^{\prime}$-AAGATCCTGACCGAGCGTGG, antisense $5^{\prime}$-CAGCAGTGTGTT GGCATAGAGG; ChAT, sense 5'-GCCACTTGCATAGGTGAGGGC, antisense 5'-GCCACTAGTCAGTTGGGC.

Immunocytochemistry. Cortical neurons were plated on coverslips precoated with poly-D-lysine. On $11 \mathrm{~d}$ in vitro (DIV), neurons were fixed with $4 \%$ paraformaldehyde for $10 \mathrm{~min}$, quenched with $100 \mathrm{~mm}$ glycine for $10 \mathrm{~min}$, and permeabilized with $0.1 \%$ Triton X-100 for $10 \mathrm{~min}$. Blocking of nonspecific sites was performed with $5 \%$ normal donkey serum and $0.1 \%$ fish gelatin in PBS for $1 \mathrm{~h}$ before incubating with primary Abs in blocking solution for $1 \mathrm{~h}$. Abs and dilutions used were as follows: MAP-2 (1:1000), GFAP (1:1000), GAD65 (1:1000), mAb299 (1:500), and mAb270 (1:500). Cells were then incubated in appropriate secondary Abs (1:1000) for $1 \mathrm{~h}$ at room temperature. In some cases, nuclei were labeled with Hoechst dye for $5 \mathrm{~min}$. Cells were mounted in ProLong gold 
and allowed to cure for $24 \mathrm{~h}$ before imaging on the Olympus DSU spinning disk (Integrated Microscopy Facility, University of Chicago, Chicago, IL). Samples prepared without primary antibody served as control for background fluorescence.

Biotinylation assays. Cell-surface proteins were biotinylated using 0.5 $\mathrm{mg} / \mathrm{ml}$ EZ-link sulfo-NHS-SS-Biotin (ThermoFisher Scientific) for 20 min on ice. The cells were washed three times with PBS followed by DMEM and maintained in DMEM for the remaining time of chase. At the end of the chase period, cells were washed two times with PBS and solubilized in lysis buffer containing $1 \%$ Triton X-100 and protease inhibitors. Lysates were incubated overnight with streptavidin agarose (EMD Chemicals). The biotinylated receptors were detected by either ${ }^{125} \mathrm{I}$-epi binding to beads or by immunoblots using subunit-specific Abs. In the instance of ${ }^{125} \mathrm{I}$-epi binding, the streptavidin agarose beads were incubated with $2.5 \mathrm{~nm}{ }^{125} \mathrm{I}$-epi for $1 \mathrm{~h}$. To determine nonspecific binding, ${ }^{125} \mathrm{I}$-epi binding was done in the presence of $1 \mathrm{~mm}$ nicotine. The beads were spun down by centrifugation at high speed for $30 \mathrm{~s}$ and washed three times with lysis buffer. The radioactivity remaining on the beads was determined using gamma counting. (PerkinElmer). When subunits where detected by immunoblots, the biotinylated proteins were eluted from streptavidin agarose beads using $2 \times$ concentration of SDS sample buffer, denatured by boiling, and subjected to SDS-PAGE. The proteins were electrotransferred to PVDF membrane (Bio-Rad) and blots were probed with Abs specific for $\alpha 4$ (6963) and $\beta 2 \mathrm{HA}$ (anti-HA).

Proteasome inhibitors. Cells stably expressing $\alpha 4 \beta 2 \mathrm{HA}$ were treated with the proteasomal inhibitors $1 \mu \mathrm{M}$ MG132, $1 \mu \mathrm{M}$ lactacystin, or $10 \mu \mathrm{M}$ nicotine for $4 \mathrm{~h}$. At the end of the incubation, cells were scraped and resuspended in PBS, and subjected to ${ }^{125} \mathrm{I}$-epi binding. A parallel set of samples treated with nicotine or MG132 were lysed in lysis buffer ( $150 \mathrm{~mm} \mathrm{NaCl}, 5 \mathrm{~mm}$ EDTA, 50 $\mathrm{mm}$ Tris, $\mathrm{pH} 7.4,0.02 \% \mathrm{NaN} 3$, plus $1 \%$ Triton $\mathrm{X}-100$ ) containing protease inhibitors ( 2 mм PMSF, 2 mм $N$-ethylmaleimide, and chymostatin, pepstatin, leupeptin, and tosyllysine chloromethyl ketone at $10 \mu \mathrm{g} / \mathrm{ml}$ ), and wholecell lysates were subjected to SDS-PAGE analysis, proteins were transferred to PVDF membranes, and Western blots were probed with $6963(\alpha 4)$, anti-HA $(\beta 2 \mathrm{HA})$, or anti-actin (loading control).

Primary cultures of cortical neurons (10 DIV) were treated with proteasomal inhibitors $1 \mu \mathrm{M} \mathrm{MG132,} 1 \mu \mathrm{M}$ lactacystin, or $1 \mu \mathrm{M}$ nicotine for $17 \mathrm{~h}$, and ${ }^{125} \mathrm{I}$-epi binding was done on intact neurons. Parallel samples treated with nicotine and MG132 were processed as described above and subjected to SDS-PAGE. Proteins were transferred to PVDF membranes followed by Western blotting using Abs $6963(\alpha 4)$ and anti-actin. Alexa Fluor anti-rabbit 647 was used to detect the $\alpha 4$ subunits and actin. The blots were scanned using the Bio-Rad pharos-FX laser scanner. The band intensities were quantified using ImageJ software (NIH).

Statistical analysis. Results are expressed as mean \pm SEM of $n$ samples. Statistical significance was assessed by a two-tailed Student's $t$ test.

\section{Results}

\section{nAChR high-affinity binding sites in live cortical neurons}

Using cultured rat cortical neurons, we measured high-affinity ${ }^{125} \mathrm{I}$-epi binding to endogenous nAChRs. Neurons, 10-12 DIV, were exposed to $1 \mu \mathrm{M}$ nicotine for $17 \mathrm{~h}$ to examine the increase or "upregulation" in ${ }^{125} \mathrm{I}$-epi binding to live neurons. Nicotine treatment caused a threefold increase in ${ }^{125} \mathrm{I}$-epi binding on average $(27.6 \pm 2.4 \mathrm{SEM} \mathrm{fmol} / \mathrm{mg}$ total protein for untreated, $70.4 \pm 4.5 \mathrm{SEM} \mathrm{fmol} / \mathrm{mg}$ total protein for nicotine treated) and varied over a range of twofold to fourfold. By varying the concentration of nicotine in the medium, we assayed the nicotine dose dependence of the upregulation of the ${ }^{125} \mathrm{I}$-epi binding for cortical neurons (Fig. $1 \mathrm{~A}$ ). An $\mathrm{EC}_{50}$ value of $92 \mathrm{nM}$ was obtained by fitting the Hill equation to the data. Using a number of different techniques, we examined the subunit composition of native nAChRs in cortical neurons. Reverse transcription PCR was used to establish which mRNA transcripts were expressed in our cultures (Fig. $1 B$ ). We detected $\alpha 3, \alpha 4, \alpha 7, \beta 2$, and $\beta 4$ subunit transcripts, but not $\alpha 2, \alpha 5, \alpha 6$, and $\beta 3$ subunit transcripts with oligonucleotide sequences already established to detect nAChR subunit transcripts (Sheffield et al., 2000; Rogers et al., 2001). $\alpha 7$ subunits primarily assemble into homomeric nAChRs that bind ${ }^{125} \mathrm{I}$ - $\alpha$-bungarotoxin, but not radiolabeled agonists such as ${ }^{125} \mathrm{I}$ epi (Clarke et al., 1985; Härfstrand et al., 1988; Pauly et al., 1989). We, therefore, assayed $\alpha 3, \alpha 4, \beta 2$, and $\beta 4$ subunits at the protein level using subunit-specific Abs for each subunit. Abs specific for each of these subunits were used to precipitate nAChRs from the lysate and ${ }^{125} \mathrm{I}$-epi binding performed on the immunoprecipitated nAChRs. The percentage of available epibatidine binding sites precipitated by each of the subunit-specific Abs was determined. $\alpha 4$ - and $\beta 2$-specific Abs precipitated a significant percentage of the available ${ }^{125} \mathrm{I}$-epi binding sites in the cortical neurons, while relatively few of the ${ }^{125} \mathrm{I}$-epi binding sites were precipitated by $\alpha 3$ - and $\beta 4$-specific Abs (Fig. 1C). Similarly using immunoblots, $\alpha 4$ subunits were immunoprecipitated by $\alpha 4$ - and $\beta 2$-specific Abs (Fig. $1 D$ ). The expression of native " $\alpha 4 \beta 2$ " receptors was further assayed using immunofluorescence microscopy (Fig. $1 E, F$ ). $\alpha 4$ - and $\beta 2$-specific Abs only stained neurons that were labeled with MAP-2 Abs and not astrocytes labeled with GFAP Abs (Fig. 1E). $\alpha 4$-specific Abs stained inhibitory neurons labeled with GAD65 Abs and other neurons presumed to be excitatory (Fig. $1 F$ ). Altogether, our results indicate that the native high-affinity ${ }^{125} \mathrm{I}$-epi binding sites that are upregulated by nicotine in the cortical neurons contain primarily $\alpha 4$ and $\beta 2$ subunits and are the " $\alpha 4 \beta 2$ " subtype.

\section{Real-time kinetics of nicotine-induced upregulation onset and reversal}

We next measured how exposure of cortical cultures to nicotine altered high-affinity ${ }^{125} \mathrm{I}$-epi binding to native $\mathrm{nAChRs}$ in real time. Cortical neuronal cultures were treated with $1 \mu \mathrm{M}$ nicotine for varying times, and ${ }^{125} \mathrm{I}$-epi binding was determined for nicotine-treated and untreated cultures at the different time points. The ratio of ${ }^{125} \mathrm{I}$-epi binding of nicotine-treated to untreated cultures or the fold-increase in ${ }^{125} \mathrm{I}$-epi binding was used as a quantitative measure of nicotine-induced upregulation. The time course of upregulation after application of nicotine is displayed in Figure $2 \mathrm{~A}$. The onset of upregulation occurred at two different rates. First, there was a fast component that saturated $4 \mathrm{~h}$ after exposure and later a slower component that increased subsequently with continued nicotine exposure. Consistent with the presence of two separate processes, the data were only well fit as the sum of two exponential functions with a fast component $(\tau=$ $1.1 \mathrm{~h})$ and a slower component $(\tau=12.5 \mathrm{~h})$.

The two components of nicotine-induced upregulation were more evident when nicotine was removed from the medium after both components of upregulation were saturated with nicotine treatments (Fig. 2 B). Nicotine withdrawal initially caused a rapid decrease in the ${ }^{125}$ I-epi binding fold-increase. This fast component was followed by a much slower decay that continued days after nicotine withdrawal (Fig. $2 \mathrm{~B}$, insert). The decay rate following nicotine withdrawal was again well fit as the sum of two separate processes. The fast component decayed with a $\tau$ value of $0.45 \mathrm{~h}$, and the slow component decayed with a $\tau$ value of $12.3 \mathrm{~d}$. The slow component of the decay of nicotine-induced upregulation after nicotine removal was not observed if cultures were exposed to nicotine for $\leq 4 \mathrm{~h}$ (Fig. $2 \mathrm{C}$ ). The kinetics of the fast component of upregulation during onset was similar to the fast decay during withdrawal, and the slow onset of upregulation was similar to the slow component of decay. These results suggest that the fast components of upregulation represent the same process either turning on during onset or turning off during reversal. They also suggest that the slow components are a second slow 
A

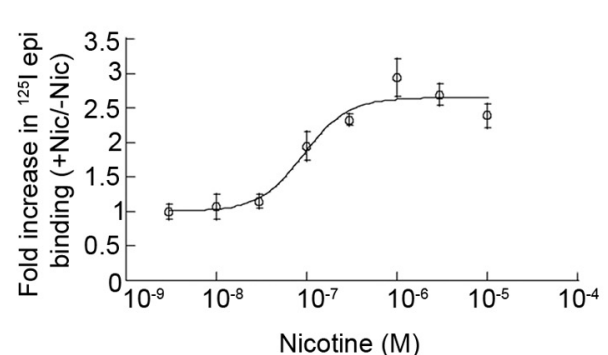

Nicotine (M)

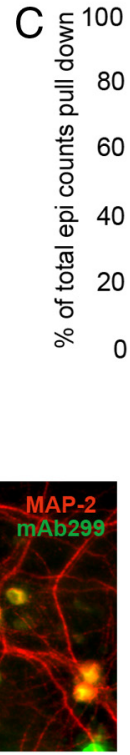

E

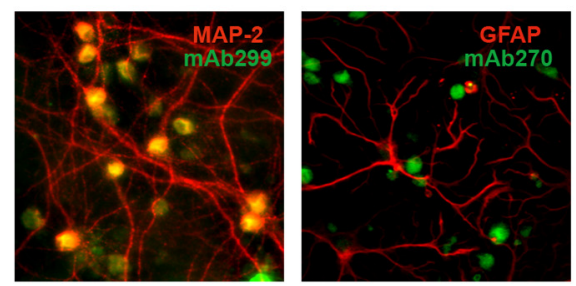

B

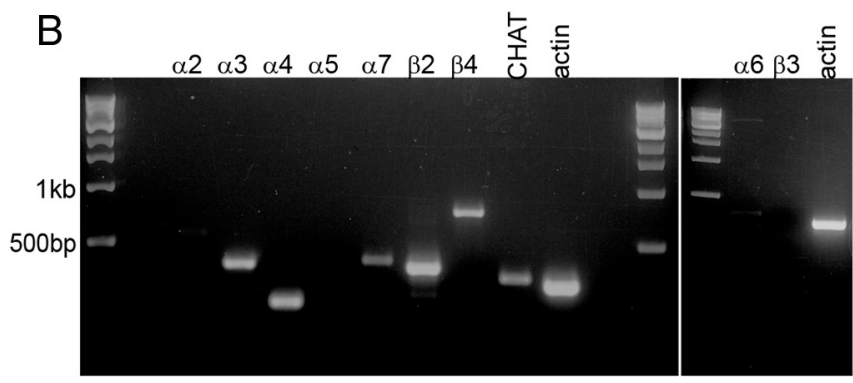

$\square-\mathrm{Nic}$

$\square+\mathrm{Nic}$

D

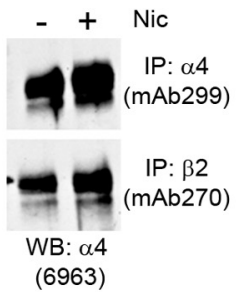

(6963)
$\mathrm{F}$

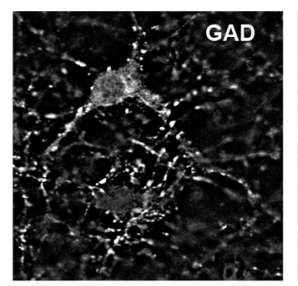

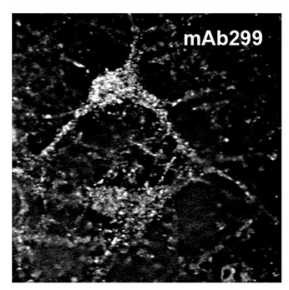

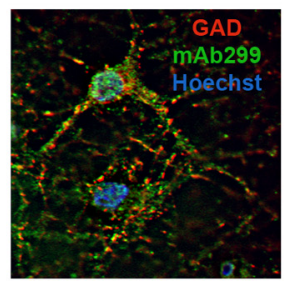

Figure 1. nAChR nicotine-induced upregulation in cortical neurons. $A$, Nicotine (Nic) dose dependence of upregulation. Primary cultures of cortical neurons (10 DIV) were treated with increasing concentrations of nicotine for $17 \mathrm{~h}$ and ${ }^{125} \mathrm{I}$-epi binding performed. Data are plotted as the average fold-increase in epibatidine binding of nicotine-treated cultures over untreated cultures \pm SEM $(n=3)$. The lines through the data represent a least-squares fit of the Hill equation to the data for cortical neurons: fold-increase $=\operatorname{Max} /\left(1+\mathrm{EC}_{50} /[\text { nicotine }]\right)^{\mathrm{n}}+1$, where Max +1 , the maximum fold-increase was 2.6; $n$, Hill coefficient was 1.8; and the $\mathrm{EC}_{50}$ value was $92 \pm 30 \mathrm{~nm}$. B, RT-PCR analysis of $\mathrm{nAChR}$ subunit transcript expression. RNA isolated from primary cortical neurons was subjected to RT-PCR analysis and run on an agarose gel, which showed the expression of the various nAChR subunit mRNAs in addition to actin and choline acetyl transferase (CHAT) as controls. A faint band was observed in the $\alpha 6$ lane, which did not correspond to the expected size of the transcript. C, Precipitation of ${ }^{125}$ I-epi binding sites from cortical neurons. Primary cultures of cortical neurons were treated with or without $1 \mu \mathrm{m}$ nicotine for $17 \mathrm{~h}$ and bound with $1 \mathrm{~nm}{ }^{125} \mathrm{I}$-epi for $20 \mathrm{~min}$ to obtain the total epibatidine sites. Parallel samples were solubilized and precipitated with subunit-specific antibodies $6963(\alpha 4), 4880(\alpha 3), \mathrm{mAb} 270(\beta 2)$, and $4886(\beta 4)$. Data are represented as the average percentage of total epibatidine binding precipitated \pm SEM $(n=3$ for 4880 and 4886; $n=5$ for 6963 and mAb270; see Fig. 6C,D for more details). D, Total $\alpha 4$ subunits immunoprecipitated (IP) by $\alpha 4$-specific antibody mAb299 or assembled $\alpha 4$ subunits coimmunoprecipitated with $\beta 2$-specific antibody mAb270 were detected on a Western blot (WB) using polyclonal anti- $\alpha 4$ antibody, 6963 . E, Cortical neurons were plated on glass coverslips and fixed, permeabilized, and costained with Abs for MAP-2 and $\alpha 4$ (mAb299) to the left or glial marker GFAP and anti- $\beta 2$ (mAb270) to the right followed by fluorescent secondary Abs. F, Cortical neurons were stained with Ab marker for inhibitory neurons (GAD65 Ab) and costained with mAb299 (anti- $\alpha 4$ ). Secondary antibody staining was done with Alexa Fluor anti-mouse 568 and anti-rat 488.

process turning on and off. In summary, we find that the nicotine-induced upregulation kinetics contains two components indicating that at least two different processes are causing upregulation. We also observed a similar biphasic decay of nicotine-induced upregulation for $\alpha 4 \beta 2$ and $\alpha 6 \beta 2$ receptors heterologously expressed in HEK 293 cells (Fig. 2D,E).

\section{The kinetics of $\mathrm{nAChR}$ degradation during nicotine withdrawal}

We began to test what mechanisms underlie the two different rates at which decay of upregulation occurred. A previous study from our laboratory found that changes in high-affinity binding with nicotine-induced upregulation did not correlate with changes in the number of $\alpha 4 \beta 2 \mathrm{nAChRs}$ stably expressed in HEK 293 cells (Vallejo et al., 2005). However, if ${ }^{125} \mathrm{I}$-epi binding is a measure of receptor numbers, then the rates of decay of upregulation with nicotine withdrawal should correlate with the rate of degradation of the mature receptor complexes that bind ${ }^{125} \mathrm{I}$-epi. To determine the rates of $\mathrm{nAChR}$ subunit assembly and degrada- tion, we used a pulse-chase approach to label the subunits with radiolabeled amino acids (Merlie and Lindstrom, 1983; Green and Claudio, 1993; Sallette et al., 2005). Unfortunately, these experiments could not be performed using cultured cortical neurons because of the limited number of neuronal cells and relatively low levels of $\mathrm{nAChR}$ expression. As an alternative, we performed pulse-chase measurements on rat $\alpha 4$ and $\beta 2$ subunits stably expressed in HEK cells that were previously used to study nicotine-induced upregulation (Vallejo et al., 2005), which also displayed fast and slow phases of the decay of upregulation following nicotine withdrawal (Fig. 2D). The subunits in the $\alpha 4 \beta 2$ stable cell line were metabolically labeled with ${ }^{35} \mathrm{~S}$-methionine/ cysteine for $15 \mathrm{~min}$. Subsequently, cells were solubilized at various chase times following nicotine withdrawal. Subunits were immunoprecipitated with several different $\alpha 4$ - and $\beta 2$-specific Abs 7-9 $\mathrm{h}$ following nicotine removal and intensities of ${ }^{35} \mathrm{~S}$ labeled bands measured. Examples of the results are displayed in Figure $3 A$. Our results were similar to previous pulse-chase analysis performed on human $\alpha 4$ and $\beta 2$ subunits transiently ex- 

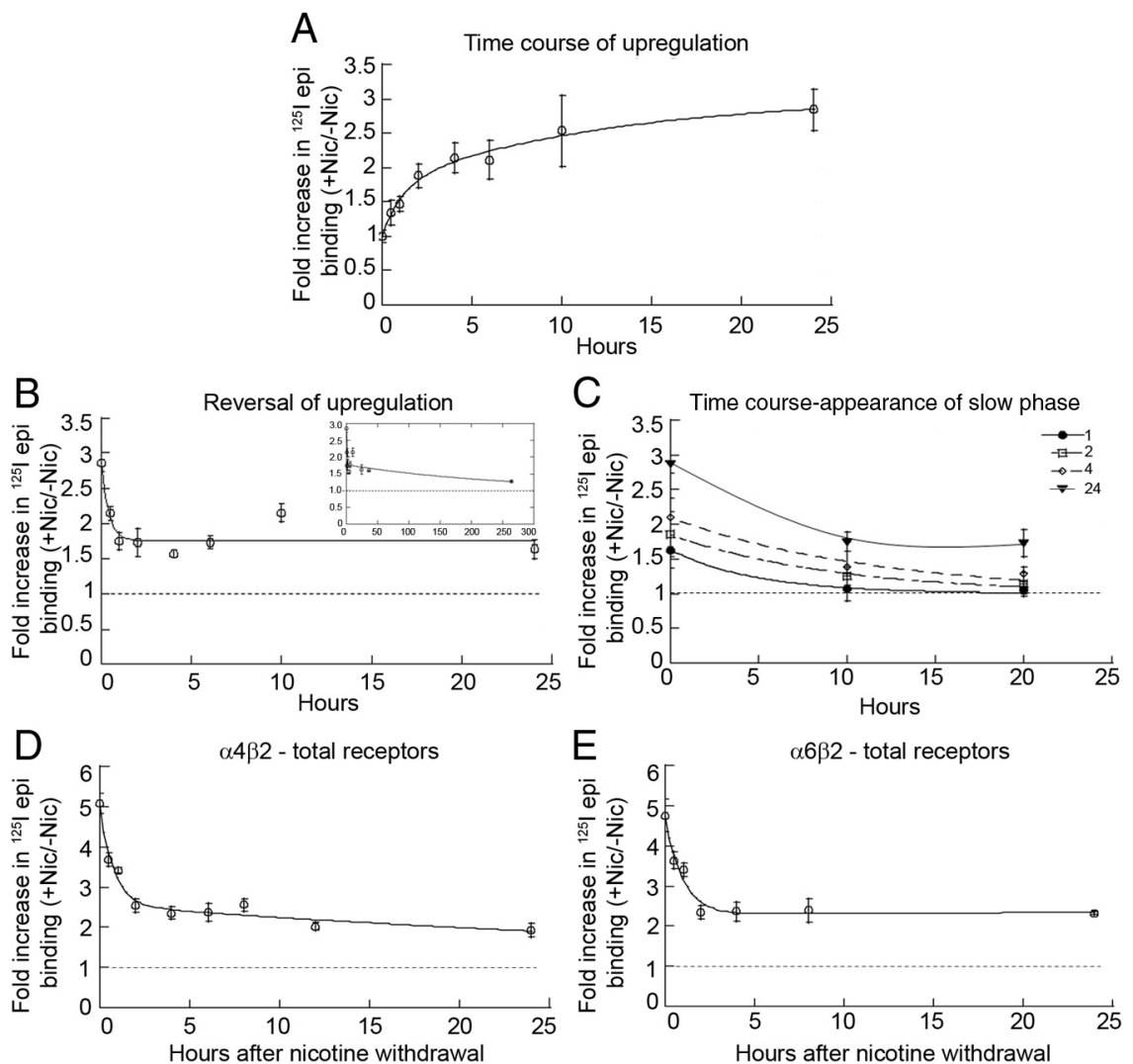

Figure 2. Upregulation onset and reversal of cortical neuron nAChRs in real time. A, Time course of upregulation. Primary cultures of cortical neurons were treated with $1 \mu \mathrm{m}$ nicotine for the indicated times. The data are plotted as the fold-increase (the ratio of nicotine-treated to untreated cultures) in ${ }^{125}$-epi binding as a function of time following nicotine exposure \pm SEM $(n=$ 5). The line represents a least-squares fit to the sum of two exponentials or fold-increase $=m 1^{*}(1-\exp (-t / \tau 1))+m 2^{*}(1-$ $\exp (-t / \tau 2))+1$ where $m 1$ and $m 2$ are the maximal values for the two exponentials $(m 1=0.89, m 2=0.74)$ and $\tau 1$ and $\tau 2$ are the respective time constants $(\tau 1=1.1 \mathrm{~h}, \tau 2=12.5 \mathrm{~h}$ ). $\boldsymbol{B}$, Decay of high-affinity nicotine-binding sites following nicotine withdrawal. Primary cultures of cortical neurons were treated with or without $1 \mu \mathrm{m}$ nicotine for $17 \mathrm{~h}$, and the cultures were washed three times with neurobasal medium and maintained in preconditioned media lacking nicotine for the indicated times. Highaffinity binding sites remaining were measured using $1 \mathrm{~nm}{ }^{125} \mathrm{I}$-epi bound for $20 \mathrm{~min}$. Displayed in the main figure are the data for the first $24 \mathrm{~h}$ after nicotine removal to show the details of the fast decay. The full time course of the decay over $11 \mathrm{~d}$ is displayed in the insert. Data are mean \pm SEM of four independent experiments performed in triplicate. The lines through the points represents the least-square fit to the data of the equation: fold increase $=m 1(\exp (-t / \tau 1))+m 2(\exp (-t / \tau 2))+1$, where $m 1$ and $m 2$ are the initial values for the two exponentials $(m 1=2.4, m 2=1.12)$ and $\tau 1$ and $\tau 2$ are the respective time constants $(\tau 1=0.45 \mathrm{~h}, \tau 2=295 \mathrm{~h})$. C, Nicotine-induced upregulation after shortexposures to nicotine. Primary cultures of cortical neurons were treated with or without $1 \mu \mathrm{m}$ nicotine for 1,2, or $4 \mathrm{~h}$ and cultures were washed and maintained in nicotine-free media as mentioned above for indicated times and ${ }^{125}$-epi binding performed. Each point is the mean $\pm \operatorname{SEM}(n=3)$. The data were fit to the equation $m(\exp -t / \tau)+1$, where $m$ is the initial value and $\tau$ is the time constant. The $\tau$ values are $0.5,0.9$, and $1.1 \mathrm{~h}$, respectively, for 1,2 , and $4 \mathrm{~h}$ time points. For comparison, the data from the $24 \mathrm{~h}$ treatment from $B$ are also displayed. $D$, HEK 293 cells stably expressing $\alpha 4 \beta 2 \mathrm{HA}$ receptors were treated with or without $10 \mu \mathrm{m}$ nicotine for $17 \mathrm{~h}$ and washed and maintained in medium without nicotine for the indicated times. High-affinity binding sites remaining were measured using 5 $\mathrm{nM}{ }^{125}$-epi bound for $20 \mathrm{~min}$. Data are mean \pm SEM $(n=5)$. The lines through the points are the least-squares fit of fold-increase $=$ $m 1(\exp (-t / \tau 1))+m 2(\exp (-t / \tau 2))+1$, where $m 1$ and $m 2$ are the initial values for the 2 exponentials $(m 1=1.3, m 2=2.5)$, and $\tau 1$ and $\tau 2$ are the respective time constants $(\tau 1=0.76 \mathrm{~h}, \tau 2=44 \mathrm{~h})$. E, HEK293 cells stably expressing $\alpha 6$ Flag $\beta 2 \mathrm{HA}$ were treated with 30 $\mu \mathrm{m}$ nicotine for $17 \mathrm{~h}$ to achieve maximum upregulation and then grown in the absence of nicotine for the indicated times. High-affinity binding sites remaining were measured using $2.5 \mathrm{nM}{ }^{125} \mathrm{I}$-epi bound for $20 \mathrm{~min}$. Data are mean $\pm \mathrm{SEM}(n=6)$. The lines were the fits to the equation in $\boldsymbol{D}(m 1=1.0, m 2=2.46 ; \tau 1=0.94 \mathrm{~h}, \tau 2=\sim 500 \mathrm{~h}$ ). Because the HEK cells stably expressing $\alpha 6 \beta 2$ receptors did not survive much beyond $48 \mathrm{~h}$ after the nicotine removal, the estimate of $\tau 2$ is an approximate. Nic, Nicotine.

pressed in HEK cells (Sallette et al., 2005). Subunit assembly as assayed by increases in subunit coprecipitation peaked over the first $60-90 \mathrm{~min}$. The peak in subunit assembly was followed by a period over the next $5-8 \mathrm{~h}$ where subunit degradation predominated, as assayed by decreases in subunit band intensities. Differences in the assembly of the subunits were observed depending on which $\mathrm{Ab}$ was used, and these differences are further described in more detail later (see Fig. 6). However, the rate of subunit degradation was independent of the $\mathrm{Ab}$ used for immunoprecipitation.

The averaged results of the ${ }^{35}$ S-labeled band intensities precipitated by the different subunit-specific Abs are displayed in Figure 3, $B$ and $C$, and were used to approximate rates of degradation of the $\alpha 4$ and $\beta 2$ subunits after nicotine withdrawal. The rates of degradation of the newly synthesized subunits were relatively fast $(\tau=3-6 \mathrm{~h}$ ), but significantly slower than the fast phase of the decay of upregulation after nicotine withdrawal $(\tau=$ $0.76 \mathrm{~h}$; see Table 1 ). The degradation rate was not significantly different whether the cells were treated or untreated with nicotine. As discussed by Sallette et al. (2005), the kinetics of $\alpha 4$ and $\beta 2$ subunit assembly and degradation in the ER are complex and are the combination of several processes including the degradation of unassembled subunits, the assembly of immature subunit complexes, and the maturation of the subunit complexes. Surprisingly, the degradation rate for the newly assembled subunits (subunits that coprecipitated) was not significantly different from the degradation rate for the total cellular pool of subunits or the rate for unassembled subunits determined by stable expression of $\alpha 4$ subunits alone (data not shown). Using the pulsechase analysis, we primarily precipitated a rapidly degrading pool of labeled subunits. The pool of mature nAChRs that is trafficked to the cell surface and degraded at a slower rate is a small proportion of the total subunit pool that is labeled during synthesis. Following loss of the rapidly degrading pool of labeled subunits, the levels of remaining subunits indicate that $<20-30 \%$ of newly synthesized subunits are further assembled into the mature pool of nAChRs (Fig. $3 B, C)$.

Next, we used cell-surface biotinylation to assay the degradation rate of mature cellsurface nAChRs after nicotine withdrawal (Fig. 3D). Cell-impermeable biotinylation reagents were used to biotinylate $n A C h R s$ on the surface of intact cells. Surface nAChRs on intact cells were briefly biotinylated with membrane-impermeable reagents at the end of the nicotine treatment $(10 \mu \mathrm{M}$ nicotine, $17 \mathrm{~h}$ ). After biotinylation and nicotine treatment, the cells were chased for the indicated times in the absence of nicotine, and subunit band intensities were determined from immunoblots of the surface-biotinylated subunits (Fig. 3D). The decay of the surface receptor $\alpha 4$ and $\beta 2$ subunit bands as a function of time after biotinylation and nicotine withdrawal in Figure $3 D$ is a measure of the rate of degradation of the surface nAChRs. We observed no significant differences in the rates of degradation for the $\alpha 4$ and $\beta 2$ subunits, whether the surface nAChRs had been nicotine pretreated or untreated. The decay of the band intensities after nicotine with- 
A Nicotine withdrawal
Control Nicotine pretreated

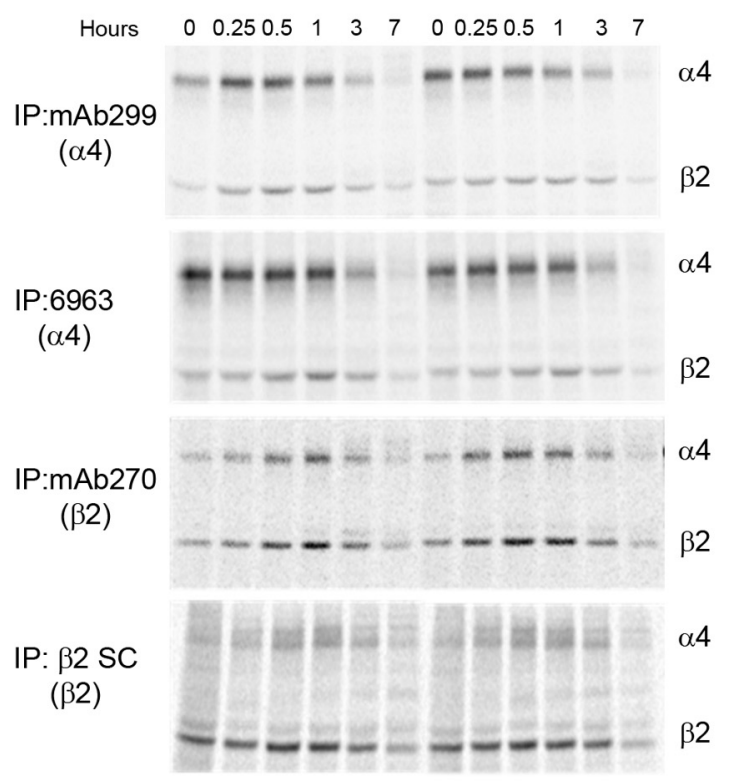

$\mathrm{D}$

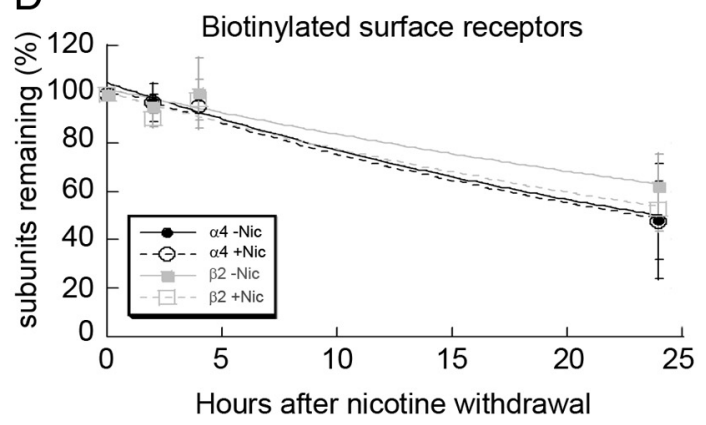

$\mathrm{B}$

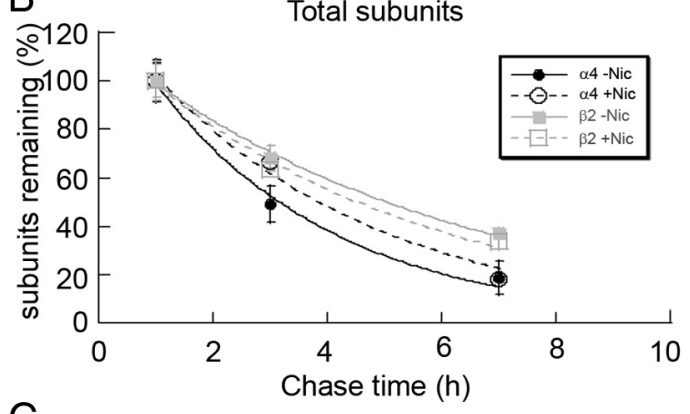

C

Assembled subunits

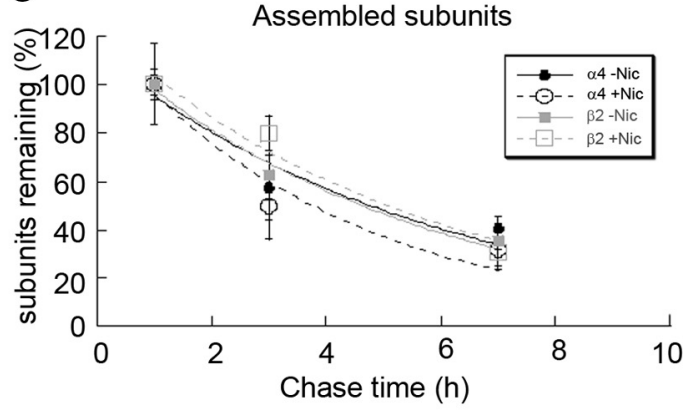

$\mathrm{E}$

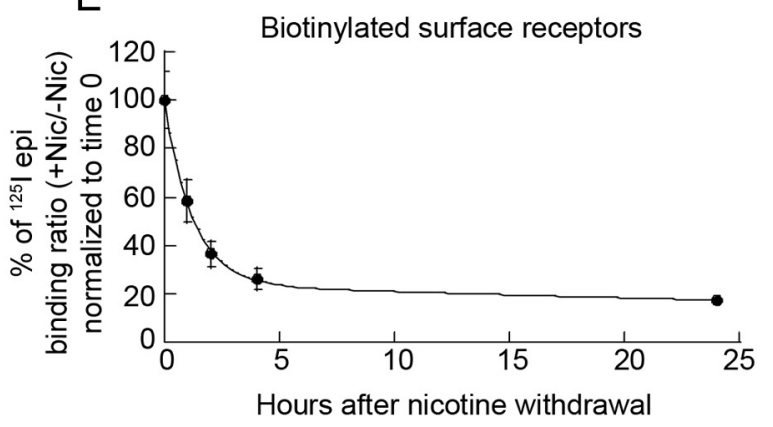

Figure 3. $\alpha 4$ and $\beta 2$ subunit degradation after nicotine (Nic) withdrawal. $\boldsymbol{A}$, Pulse-chase results for ${ }^{35}$ S-labeled subunits precipitated with different $\alpha 4$ - and $\beta 2$-specific Abs. Cell line stably expressing $\alpha 4 \beta 2$ receptors was treated with $10 \mu \mathrm{m}$ nicotine for $17 \mathrm{~h}$ to achieve maximum upregulation. The cells were metabolically labeled with ${ }^{35} \mathrm{~S}$-met/cys for $15 \mathrm{~min}$ and chased in the absence of nicotine (withdrawal). Subunits were immunoprecipitated (IP) with $\alpha 4$-specific Abs, polyclonal 6963 or mAb299 or $\beta 2$-specific Abs, mAb270 or polyclonal anti- $\beta 2$ (SC), and subjected to SDS-PAGE. B, C, Degradation of newly synthesized subunits after nicotine withdrawal. Band intensities in $A$ are plotted as a function of the chase time and are the mean \pm SEM $(n=3)$ for $\alpha 4$ or $\beta 2$ subunits precipitated by both Abs. Only the values beginning at the $1 \mathrm{~h}$ time point are displayed, the time when the band intensities began to decay. Displayed in $\boldsymbol{B}$ are the values for the newly synthesized $\alpha 4$ or $\beta 2$ subunits immunoprecipitated by the Abs ("Total" subunits). The data were fit to single exponential equations: percentage of subunits remaining $=100 \%$ (exp $(-t / \tau)$ ) where $\tau$ is the characteristic decay rate. The $\tau$ values were $3.2 \mathrm{~h}$ for $\alpha 4$ subunits and $5.9 \mathrm{~h}$ for $\beta 2$ subunits for no nicotine treatment and $3.9 \mathrm{~h}$ for $\alpha 4$ subunits and $5.2 \mathrm{~h}$ for $\beta 2 \mathrm{subunits}$ for nicotine pretreatment. Displayed in Care the values for $\alpha 4$ or $\beta 2$ subunits that coimmunoprecipitated with the precipitated subunits ("Assembled" subunits). The $\tau$ values were $5.8 \mathrm{~h}$ for $\alpha 4$ subunits and $5.3 \mathrm{~h}$ for $\beta 2$ subunits for no nicotine treatment, and $4.4 \mathrm{~h}$ for $\alpha 4$ subunits and $5.6 \mathrm{~h}$ for $\beta 2$ subunits for nicotine pretreatment. D, Degradation of surface $\alpha 4 \beta 2 \mathrm{nAChRs}$ after nicotine withdrawal. Cells expressing $\alpha 4 \beta 2 \mathrm{nAChRs}$ were treated with or without $10 \mu \mathrm{m}$ nicotine for $17 \mathrm{~h}$. Surface receptors were biotinylated and maintained at $37^{\circ} \mathrm{C}$ in the absence of nicotine for indicated times. Cells were lysed and biotinylated surface nAChRs precipitated with streptavidin agarose and analyzed by SDS-PAGE and immunoblots. Subunits on immunoblots were detected using $\alpha 4$-specific (polyclonal 6963) or $\beta 2$-specific (anti-HA) Abs. The intensity of subunits remaining at each time point was plotted as a function of time \pm SEM $(n=3)$. The lines through the data points represent fits of the equation to the data: percentage of subunits remaining $=m(\exp -t / \tau)$, where $m$ is the initial value and $\tau$ is the decay rate. The $\tau$ values in untreated cells were $32.5 \mathrm{~h}$ for $\alpha 4$ and $49.5 \mathrm{~h}$ for $\beta 2$ and that of nicotine pretreated cells were $32.2 \mathrm{~h}$ for $\alpha 4$ and $38.2 \mathrm{~h}$ for $\beta 2$.E, Decay of ${ }^{125} \mathrm{I}$-epi binding to cell surface receptors following nicotine withdrawal. Cells expressing $\alpha 4 \beta 2 \mathrm{nAChRs}$ were upregulated with $10 \mu \mathrm{m}$ nicotine for $17 \mathrm{~h}$ or left untreated and subsequently surface biotinylated and lysed at the indicated times. Surface nAChRs were precipitated with streptavidin agarose after ${ }^{125} \mathrm{I}$-epi binding. The data are plotted as the ratio of precipitated ${ }^{125} \mathrm{I}$-epi counts for nicotine pretreated samples to untreated samples and displayed as the percentage of the initial value. The points and error bars are the mean $\pm \operatorname{SEM}(n=3)$. The lines through the data points are least-squares fits of the equation to the data: percentage of initial value $=m 1(\exp (-t / \tau 1))+m 2$ $(\exp (-t / \tau 2))+1$, where $m 1$ and $m 2$ are the initial values $(m 1=81 \%, m 2=19 \%)$ and $\tau 1$ and $\tau 2$ are the respective time constants $(\tau 1=1.2 \mathrm{~h}, \tau 2=45 \mathrm{~h})$.

drawal was well fit as a single exponential process with a mean $\tau$ of $35 \mathrm{~h}$. Thus, mature $\alpha 4 \beta 2 \mathrm{nAChRs}$ are degraded at rates much slower than the newly assembled receptor subunits assayed in Figure 3, $B$ and $C$, consistent with the degradation rates that had been previously measured for $\alpha 4 \beta 2 \mathrm{nAChRs}$ (Darsow et al., 2005) and other nAChRs (Christianson and Green, 2004).

We also examined the kinetics of ${ }^{125} \mathrm{I}$-epi binding to the plasma membrane pool of $\alpha 4 \beta 2$ receptors after nicotine withdrawal. To do this, ${ }^{125} \mathrm{I}$-epi binding was performed on the solu- bilized, surface-biotinylated $\alpha 4 \beta 2 \mathrm{nAChRs}$ as a function of time after nicotine withdrawal (Fig. 3E). The reversal of ${ }^{125} \mathrm{I}$-epi binding to the surface-biotinylated nAChRs displayed both the fast and slow components similar to that observed when the ${ }^{125} \mathrm{I}$-epi binding was done on the whole-cell population of nAChRs (Fig. $2 B, D)$. Because the fast component was not observed when the degradation of surface nAChRs was assayed (Fig. 3D), the ${ }^{125} \mathrm{I}-$ epi binding that rapidly reverses after nicotine withdrawal in Figure $3 E$ cannot be caused by surface $n A C h R$ degradation or any 
Table 1. Parameters for the nicotine-dose dependence ( $\mathrm{EC}_{50}$ values) and kinetics ( $\tau$ values) for upregulation (onset and reversal) of native and heterologously expressed $\mathrm{nAChR}$ subtypes obtained from the relevant equation fit to the data (see Figs. $1 B, 2 A, B$ legends)

\begin{tabular}{|c|c|c|c|c|c|}
\hline & \multirow{2}{*}{$\begin{array}{l}\text { Nicotine dose } \\
\text { dependence }\left(\mathrm{EC}_{50}\right)\end{array}$} & \multicolumn{2}{|c|}{$\begin{array}{l}\text { Upregulation onset } \\
\text { with nicotine } \\
\text { addition }\end{array}$} & \multicolumn{2}{|c|}{$\begin{array}{l}\text { Upregulation } \\
\text { reversal with } \\
\text { nicotine removal }\end{array}$} \\
\hline & & $\tau 1(\mathrm{~h})$ & $\tau 2(\mathrm{~h})$ & $\tau 1(\mathrm{~h})$ & $\tau 2(\mathrm{~h})$ \\
\hline$\alpha 4 \beta 2$ & ${ }^{a} 2.7 \times 10^{-7}$ & & $14.5^{a}$ & 0.76 & 44 \\
\hline Surface & & & $\sim 15^{a}$ & 1.2 & 45 \\
\hline$\alpha 6 \beta 2$ & ${ }^{b} 4.6 \times 10^{-5}$ & $b_{1.2}$ & ND & 0.94 & $\sim 500$ \\
\hline$\alpha 3 \beta 2$ & ${ }^{b} 1.3 \times 10^{-4}$ & ${ }^{b} 1.5$ & ND & ND & ND \\
\hline Cortical neurons & $9.2 \times 10^{-8}$ & 1.1 & 12.5 & 0.45 & 295 \\
\hline
\end{tabular}

Dose dependence of upregulation was measured by treating the HEK cells expressing nAChR subtypes or primary cultures of cortical neurons with increasing concentrations of nicotine and measuring increases in ${ }^{125}$-epi binding. Onset of upregulation was measured by treating cells with appropriate concentrations of nicotine as determined through dose dependence and measuring increases in ${ }^{125} \mathrm{I}$-epi binding as a function of time. Reversal of upregulation was initiated by treating the cells with appropriate concentrations of nicotine that cause maximum upregulation, washing the cells to remove nicotine, maintaining them in nicotine-free media, and measuring the ${ }^{125}$-epi binding as a function of time. ND, Not determined.

${ }^{a}$ Taken from Vallejo et al. (2005).

${ }^{b}$ Taken from Walsh et al. (2008). drawal and, thus, did not observe the second component of upregulation. We find that the slow decay rate of the second upregulation component ( $\tau=45 \mathrm{~h}$; see Table 1$)$, unlike the first, does correlate with the rate of degradation of the surface nAChRs (mean $\tau=35 \mathrm{~h}$ ). These results demonstrate that a second set of mechanisms, which changes the number of nAChRs, cause the slow component of nicotine-induced upregulation.

\section{Kinetics of degradation of $\mathbf{n A C h R s ~ d u r i n g ~ n i c o t i n e ~ e x p o s u r e ~}$}

During nicotine withdrawal, the degradation rate of newly synthesized subunits did not differ between cells that were nicotine pretreated or left untreated (Fig. $3 A-C$ ). However, when nicotine treatment was maintained ( $10 \mu \mathrm{M}$ nicotine for $17 \mathrm{~h}$ ), we observed significant differences in the degradation rate for newly synthesized $\beta 2$ subunits in the total and assembled pool, but less so for $\alpha 4$ subunits (Fig. $4 A-C$ ). The degradation rate of mature nAChRs on the cell surface was not altered by nicotine treatment as shown previously (Darsow et al., 2005; Vallejo et al., 2005). Thus, maintained exposure to nicotine slows the degradation rate of newly synthesized subunits, largely $\beta 2$ subunits (Fig. $4 B$ ), leading to increased assembly of receptors.
A

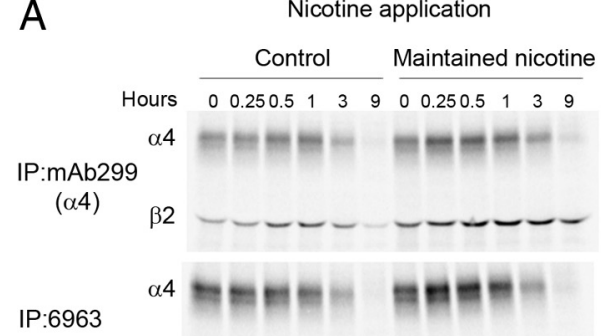

$(\alpha 4)$

$\beta 2$

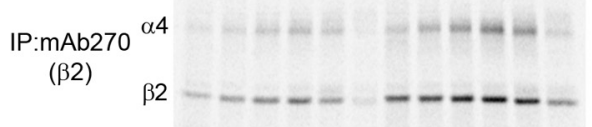

IP: $\beta 2$ SC $\alpha^{4}$

$(\beta 2)$

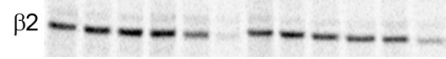

$\mathrm{B}$

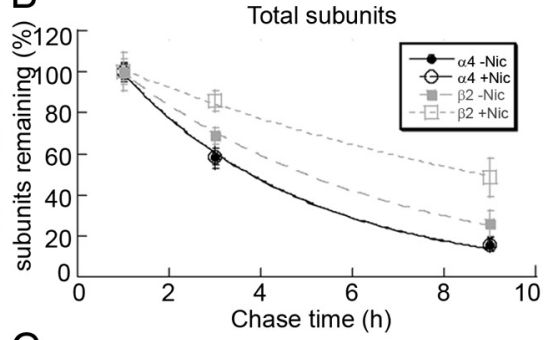

C

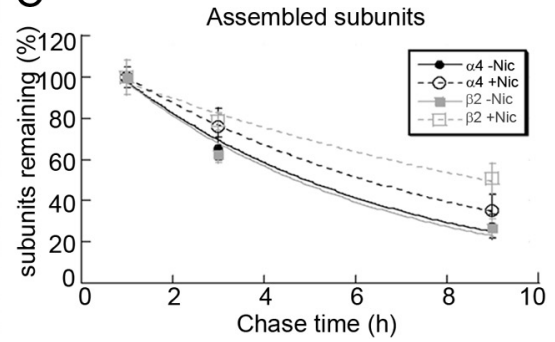

Figure 4. $\quad \alpha 4$ and $\beta 2$ subunit degradation during nicotine (Nic) exposure. $\boldsymbol{A}$, Pulse-chase results for ${ }^{35} \mathrm{~S}$-labeled subunits precipitated with different $\alpha 4$ - and $\beta 2$-specific Abs during sustained nicotine exposure. Cell line stably expressing $\alpha 4 \beta 2$ receptors was treated with $10 \mu \mathrm{m}$ nicotine for $17 \mathrm{~h}$ to achieve maximum upregulation. The cells were metabolically labeled with ${ }^{35} \mathrm{~S}$-met/cys for $15 \mathrm{~min}$ and chased in the presence of nicotine (upregulation). Subunits were immunoprecipitated (IP) with $\alpha 4$-specific antibodies, polyclonal 6963 or mAb299 or $\beta 2$-specific Abs, or mAb270 or polyclonal anti- $\beta 2$ (SC), and subjected to SDS-PAGE. B,C, Degradation of newly synthesized subunits during nicotine exposure. $\alpha 4 \beta 2$-expressing HEK cells were treated as in Figure $3 A-C$, but instead newly synthesized subunits were chased in culture for the indicated time intervals in the presence or absence of nicotine. Cells were lysed and immunoprecipitated with the Abs described. Band intensities are plotted as a function of the chase time and are the mean $\pm \operatorname{SEM}(n=3)$ for $\alpha 4$ or $\beta 2$ subunits precipitated by both Abs. Only the values beginning at the $1 \mathrm{~h}$ time point are displayed, the time when the band intensities began to decay. Displayed in $\boldsymbol{B}$ are the values for the newly synthesized $\alpha 4$ or $\beta 2$ subunits immunoprecipitated by the Abs ("Total" subunits). The data were fit to single exponential equations: percentage of subunits remaining $=100 \%(\exp (-t / \tau))$ where $\tau$ is the characteristic decay rate. The $\tau$ values for untreated cells were $4.0 \mathrm{~h}$ for $\alpha 4$ and $5.8 \mathrm{~h}$ for $\beta 2$ and $4.0 \mathrm{~h}$ for $\alpha 4$ and $11.2 \mathrm{~h}$ for $\beta 2$ for nicotine-treated cells. Displayed in $C$ are the values for the newly synthesized $\alpha 4$ or $\beta 2$ subunits that coimmunoprecipitated with the precipitated subunits ("Assembled" subunits). The $\tau$ values for untreated cells were $5.8 \mathrm{~h}$ for $\alpha 4$ and $5.5 \mathrm{~h}$ for $\beta 2$ and $7.6 \mathrm{~h}$ for $\alpha 4$ and $11.7 \mathrm{~h}$ for $\beta 2$ for nicotine-treated cells.

change in surface nAChR numbers. These findings regarding the rapid phase of nicotine-induced upregulation are in agreement with our previous conclusions about mechanisms underlying nicotine-induced upregulation for $\alpha 4 \beta 2 \mathrm{nAChRs}$ stably expressed in HEK 293 cells (Vallejo et al., 2005). However, in the previous study, we did not measure the effects of nicotine with-

\section{Proteasome-mediated degradation of nAChR subunits causes the slow component of upregulation}

We next examined the mechanisms causing the slow phase of nicotine-induced upregulation. Degradation of the newly synthesized subunits is likely occurring in the ER, similar to other nAChRs (Christianson and Green, 2004; Grailhe et al., 2004). We had found that ER-associated degradation (ERAD) of the nAChR subunit was mediated by proteasomes, as evidenced by increases in subunit levels and their assembly in the ER after blocking proteasome activity (Christianson and Green, 2004). To similarly test whether a loss of proteasome activity results in increases in $\alpha 4$ and $\beta 2$ subunit levels in the $\mathrm{ER}$, we compared the effects of the proteasome inhibitor, MG132, and nicotine treatment on the levels of $\alpha 4$ and $\beta 2$ in the $\alpha 4 \beta 2$-expressing HEK cell line. Treatment of the cells with MG132 caused a twofold increase in the steady-state levels of both $\alpha 4$ and $\beta 2$ subunits (Fig. 5A, left, middle left, middle right). Nicotine treatment resulted in a twofold increase in $\beta 2$ subunit steady-state levels (Fig. $5 \mathrm{~A}$, left, middle right) but had a much smaller effect on $\alpha 4$ subunit levels (1.3-fold; Fig. 5A, left, middle left), consistent with the greater effect nicotine had on the rate of $\beta 2$ subunit degradation compared with $\alpha 4$ subunit degradation (Fig. $4 B, C$ ). In contrast to a previous study, which suggested that nicotine directly inhibits proteasomal activity (Rezvani et al., 2007), we observe a differential effect of nicotine treatment on $\alpha 4$ and $\beta 2$ subunit levels compared with the proteasomal block suggesting that nicotine is not directly inhibiting proteasomal activity in the conditions measured in this study. We obtained additional evidence that nicotine is not directly inhib- 

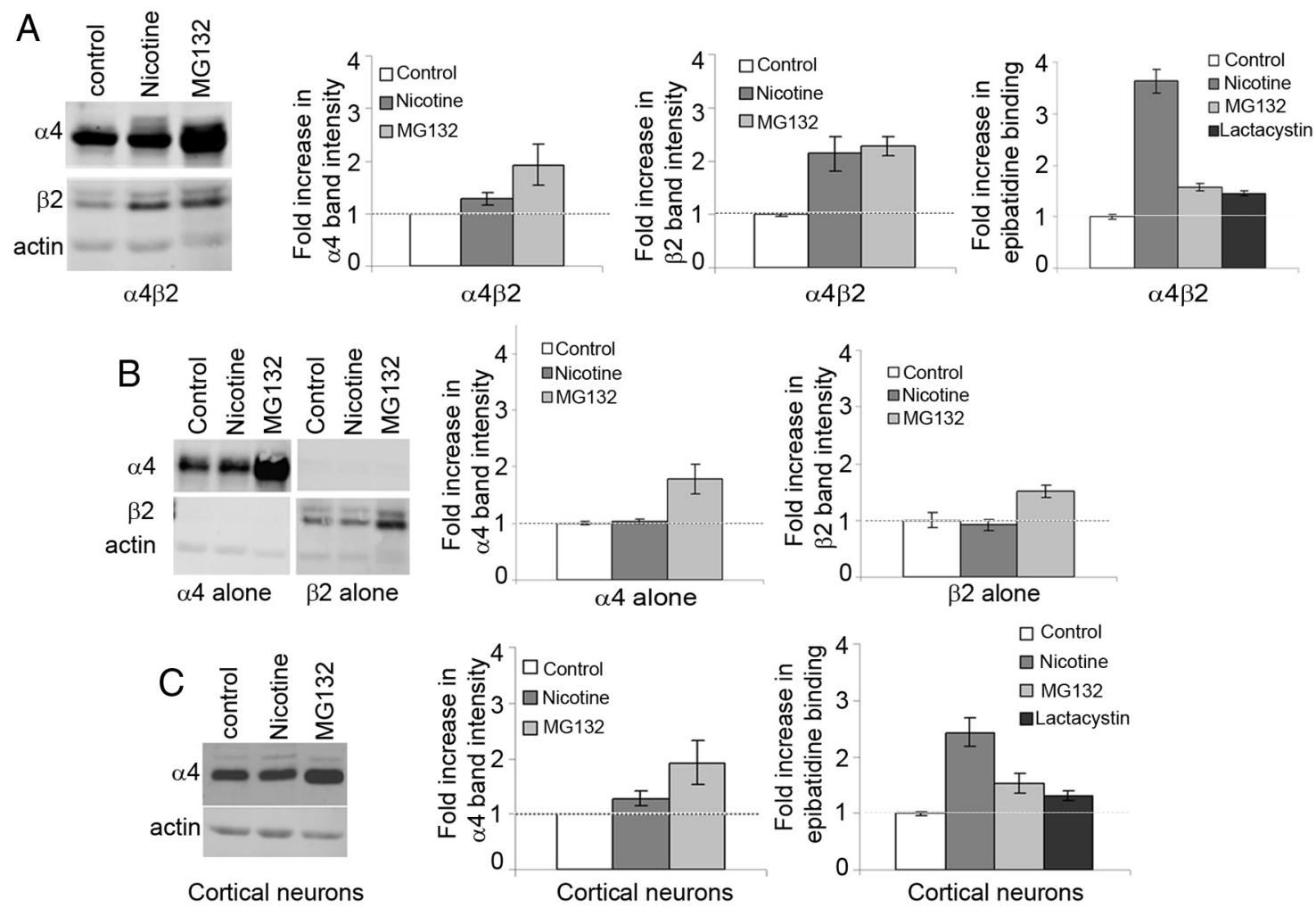

Figure 5. Nicotine slows proteasomal degradation of $\alpha 4$ and $\beta 2$ subunit. $A$, Effects of nicotine and proteasome inhibitors on heterologously expressed $\alpha 4 \beta 2 \mathrm{HA}$ receptors. Left, $\alpha 4 \beta 2 \mathrm{HA}$ expressing HEK cells were treated with $10 \mu \mathrm{m}$ nicotine, $1 \mu \mathrm{m}$ MG132, or vehicle for $4 \mathrm{~h}$. Whole-cell lysates from treated cells were subjected to SDS-PAGE, and proteins were transferred to PVDF and immunoblotted with $6963(\alpha 4)$ or anti-HA ( $\beta 2 \mathrm{HA})$ or anti-actin (loading control). Middle left, Quantification of $\alpha 4$ subunit band intensity normalized to control levels shown in the first panel \pm SEM $(n=3)$. Middle right, Quantification of $\beta 2$ subunit band intensity normalized to control levels as shown in the left panel \pm SEM $(n=3)$. Right, ${ }^{125}$ l-epi binding was performed on cells treated with $10 \mu \mathrm{m}$ nicotine, $1 \mu \mathrm{m} \mathrm{Mg132}$, or $1 \mu \mathrm{m}$ lactacystin. The fold-increase in ${ }^{125}$-epi binding observed with nicotine or proteasome blockers MG132 or lactacystin over nontreated control are plotted \pm SEM $(n=4)$. B Effects of nicotine and proteasome inhibitors on $\alpha 4$ and $\beta 2$ subunits expressed alone. Left, HEK cells stably expressing either $\alpha 4$ or $\beta 2$ subunit alone were treated with or without $10 \mu \mathrm{m}$ nicotine or $1 \mu \mathrm{m}$ MG132 for $4 \mathrm{~h}$. Whole-cell lysates were subjected to SDS-PAGE and immunoblotted with anti $\alpha 4$ (6963) and anti-HA ( $\beta 2)$ Abs. Middle, Quantification of band intensity of $\alpha 4$ subunit following nicotine or MG132 treatment normalized to control levels in left panel \pm SEM $(n=3)$. Right, Quantification of band intensity of $\beta 2$ subunits following nicotine or MG132 treatment normalized to control levels in, first panel $\pm \operatorname{SEM}(n=3)$. C, Effects of nicotine and proteasome inhibitors on endogenous nicotinic receptors expressed in cortical neurons. Left, Primary cultures of cortical neurons (10 DIV) were treated with $1 \mu \mathrm{m}$ nicotine, $1 \mu \mathrm{m}$ MG132, or vehicle for $17 \mathrm{~h}$. Lysates (from two $6 \mathrm{~cm}$ plates) were loaded on SDS-PAGE, and proteins were transferred to PVDF and immunoblotted with $6963(\alpha 4)$ or anti-actin (loading control). Middle, Quantification of band intensity of $\alpha 4$ subunit normalized to control levels in the left panel \pm SEM $(n=3)$. Right, ${ }^{125}$-epi binding was performed on primary cultures of cortical neurons treated with $1 \mu \mathrm{m}$ nicotine, $1 \mu \mathrm{M}$ MG132, or $1 \mu \mathrm{m}$ lactacystin as described above \pm SEM $(n=3)$.

iting proteasomes when $\alpha 4$ and $\beta 2$ subunits were stably expressed individually in HEK cells (Fig. 5B). Proteasomal inhibition still increased $\alpha 4$ and $\beta 2$ subunit levels twofold, but nicotine treatment had no effect on $\alpha 4$ and $\beta 2$ subunit levels when expressed individually (Fig. $5 B$, left, middle, right). These results suggested that the nicotine-induced effects on $\alpha 4$ and $\beta 2$ subunit levels and degradation rates require the presence of both subunits and subunit assembly in cells.

Inhibition of proteasomes had similar effects on nicotineinduced upregulation of $\alpha 4 \beta 2 \mathrm{nAChRs}$ in cortical neurons. Only $\alpha 4$ subunit levels could be measured using immunoblots for cortical neurons. Similar to the $\alpha 4 \beta 2$ HEK cell line proteasomal inhibition and nicotine-induced upregulation increased $\alpha 4$ subunit levels, a 1.7-fold increase with MG132 treatment and a 1.2fold increase with nicotine treatment (Fig. 5C, middle). A significantly smaller fold-increase in ${ }^{125} \mathrm{I}$-epi binding was observed with protease inhibitors (MG132 and lactacystin) than for nicotine-induced upregulation for both cortical neurons (Fig. $5 C$, right) and $\alpha 4 \beta 2$-expressing cells (Fig. $5 A$, right) even though proteasomal inhibition increased subunit levels to a greater extent. Thus, it appears that nicotine binding to pre-existing $n A C h R s$ induces a decrease in proteasome-mediated degradation of $\mathrm{nAChR}$ subunits, primarily $\beta 2$ subunits, during ERAD. Slow- ing of $\beta 2$ subunit ERAD increases the number of subunits that assemble into nAChRs in the ER. Increased nAChR assembly, in turn, increases $\mathrm{nAChR}$ numbers and ${ }^{125} \mathrm{I}$-epi binding at intracellular and surface sites, similar to what was found previously with muscle nAChR subunits when ERAD was blocked (Christianson and Green, 2004).

\section{Nicotine-induced $n A C h R$ conformational changes occur during the fast phase of upregulation}

We also investigated the mechanisms responsible for the fast component of nicotine-induced upregulation that occur following nicotine withdrawal. In Figure 3, we found that the fast decay of nicotine-induced upregulation during nicotine withdrawal does not correspond to a change in the number of nAChRs. If not a change in the number of nAChRs, what causes the decay in ${ }^{125} \mathrm{I}$-epi binding? We had previously proposed that nicotine induces a conformational change during upregulation causing nAChRs to transition from a low-affinity resting state to a highaffinity upregulated state (Vallejo et al., 2005). Evidence of this conformational change during upregulation was a change in the $\alpha 4 \beta 2$ nAChR functional state with upregulation (Buisson and Bertrand, 2001; Vallejo et al., 2005). Another assay of nAChR conformational changes is the interaction between nAChRs 

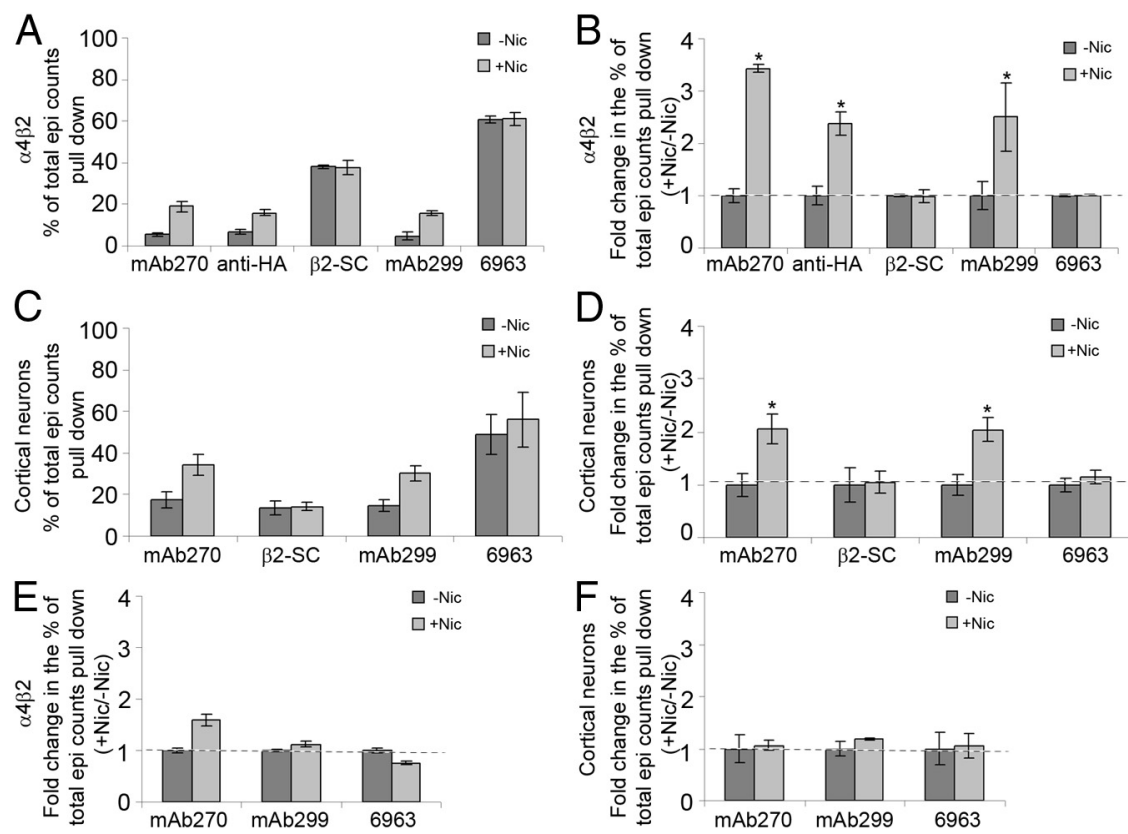

Figure 6. Nicotine alters binding of nAChR conformation-dependent Abs. $\boldsymbol{A}$, Immunoprecipitation of ${ }^{125}$ I-epi binding sites from HEK cells stably expressing $\alpha 4 \beta 2 \mathrm{HA}$ receptors. ${ }^{125}$-epi binding was performed on cells treated with or without $10 \mu \mathrm{m}$ nicotine for $17 \mathrm{~h}$ to obtain total binding. A parallel set of cells was solubilized and nAChRs immunoprecipitated with $\alpha 4$ (mAb299 or 6963 polyclonal anti- $\alpha 4$ ) or $\beta 2$ [mAb270, polyclonal anti- $\beta 2$ ( $\beta 2$ SC), or anti-HA] subunit-specific Abs and bound with ${ }^{125}$ I-epi. Data points are the average percentage of total binding precipitated \pm SEM $(n=5)$. $\boldsymbol{B}$, Data in Fig. $5 A$ are replotted as fold-change or ratio (nicotine-treated to untreated samples) of the percentage of total ${ }^{125} \mathrm{I}$-epi counts pulled down by each of the Abs in $\boldsymbol{A}$. $\mathrm{mAb270}$, anti-HA mAb, and mAb299 precipitated a significantly larger percentage of total epibatidine counts in $\alpha 4 \beta 2$ samples exposed to nicotine ( $t$ test; ${ }^{*} p<0.05$ ), whereas polyclonal antibodies $6963(\alpha 4)$ and anti- $\beta 2$ (SC) precipitated a similar percentage of total ${ }^{125}$ I-epi counts from nicotine-treated and nontreated cells. $\boldsymbol{C}$, Immunoprecipitation of ${ }^{125}$-epi binding sites from cortical neurons. Primary cultures of cortical neurons (10 DIV) were treated with or without $1 \mu \mathrm{m}$ nicotine for $17 \mathrm{~h}$, and bound with ${ }^{125} \mathrm{I}$-epi to obtain the total number of ${ }^{125} \mathrm{I}$-epi sites. Parallel samples were lysed and precipitated with subunit-specific antibodies as in $\boldsymbol{A}$. Data are represented as the average percentage of total binding precipitated $\pm \operatorname{SEM}(n=5)$. Similar to the cell line expressing $\alpha 4 \beta 2 \mathrm{HA}$, mAb270 and mAb299 precipitated a significantly larger percentage of total epibatidine counts in cortical neurons exposed to nicotine $\left(t\right.$ test; $\left.{ }^{*} p<0.05\right)$. $\boldsymbol{D}$, Data in $\boldsymbol{C}$ are replotted as the ratio of the percentage of total ${ }^{125}$ I-epi counts pulled down from nicotine-treated to untreated samples by each of the Abs used. $\boldsymbol{E}$, HEK cells stably expressing $\alpha 4 \beta 2 \mathrm{HA}$ were treated with $10 \mu \mathrm{m}$ nicotine for $17 \mathrm{~h}$ and washed and maintained in nicotine-free media for $3 \mathrm{~h} .{ }^{125} \mathrm{I}$-epi binding was performed on intact cells to obtain total ${ }^{125} \mathrm{I}$-epi binding sites. Lysates were made from parallel samples, immunoprecipitated with indicated antibodies, bound with ${ }^{125} \mathrm{I}$-epi, and data plotted as in Fig. 5B. F, Cortical neurons were treated with $1 \mu \mathrm{m}$ nicotine for $17 \mathrm{~h}$ and washed and maintained in nicotine-free media for $5 \mathrm{~h}$. ${ }^{125} \mathrm{I}$-epi binding was assayed on intact neurons to obtain the total ${ }^{125} \mathrm{I}$-epi binding sites. Parallel samples were lysed and immunoprecipitated with indicated antibodies, bound with ${ }^{125}$-epi, and data plotted as in Fig. $50 \pm$ SEM $(n=3)$.

and monoclonal Abs (mAbs) with conformational-dependent epitopes. Conformational-dependent mAbs have been used to assay for muscle-type nAChR subunit conformational changes that occur during nAChR assembly and maturation in the ER (Merlie and Lindstrom, 1983; Green and Claudio, 1993).

We tested a number of $\alpha 4$ - and $\beta 2$-specific Abs to examine whether their associations with mature ${ }^{125} \mathrm{I}$-epi-bound $\alpha 4 \beta 2$ nAChRs were altered by nicotine treatment. To assay for changes in $\mathrm{Ab}-\mathrm{nAChR}$ associations caused by nicotine treatment, we measured the percentage of ${ }^{125} \mathrm{I}$-epi binding that was precipitated by each $\mathrm{Ab}$ for nicotine-treated and untreated cells. This percentage was obtained from the ratio of Ab-precipitated ${ }^{125} \mathrm{I}$-epi binding counts to total cellular ${ }^{125} \mathrm{I}$-epi binding for intact cells that were either nicotine treated or untreated. Using the stable $\alpha 4 \beta 2$ expressing cell line (Fig. 6A), two of the Abs tested showed no change in the precipitation of ${ }^{125} \mathrm{I}$-epi-bound nAChRs with nicotine treatment. The polyclonal Abs tested precipitated a higher percentage of the total ${ }^{125}$ I-epi binding counts. Three of the Abs tested showed large increases in the precipitation of ${ }^{125} \mathrm{I}$-epibound nAChRs with nicotine treatment, but precipitated a smaller percentage of the total ${ }^{125} \mathrm{I}$-epi binding counts even for the nicotinetreated cells. Two of the Abs that showed nicotine-induced changes in the precipitation of ${ }^{125}$ I-epi-bound nAChRs, $\beta 2$ specific $\mathrm{mAb} 270$, and $\alpha 4$-specific $\mathrm{mAb}$ 299 were mAbs whose interactions with nAChRs were previously shown to be dependent on subunit conformation (Harkness and Millar, 2002). These findings indicate that $\mathrm{mAb} 270$ and 299 epitopes become more accessible for binding after nicotine treatment while the availability of the $\alpha 4$ - and $\beta 2$-specific polyclonal $\mathrm{Ab}$ epitopes does not change after nicotine treatment. Surprisingly, a third Ab, specific for the HA epitope that was fused to the $\beta 2$ subunit $\mathrm{C}$ terminus, also showed similar increases in the precipitation of ${ }^{125} \mathrm{I}$-epi-bound nAChRs with nicotine treatment, suggesting that the HA epitope was changing with nicotine treatment becoming more accessible for $\mathrm{Ab}$ binding similar to mAbs 270 and 299.

We performed the same experiments with cortical neurons (Fig. 6C,D) and observed similar results. The HA-specific $\mathrm{mAb}$ could not be used because the native $\beta 2$ subunits lack the HA epitope. The $\alpha 4$ - and $\beta 2$-specific polyclonal Abs precipitated the same percentage of ${ }^{125}$ I-epibound nAChRs whether the neurons had been nicotine treated or untreated (Fig. $6 C, D)$. The $\beta 2$-specific polyclonal Ab precipitated a smaller percentage of the ${ }^{125} \mathrm{I}$ epi bound nAChRs compared with the $\alpha 4 \beta 2$ cell line. The smaller percentage resulted from a new lot of $\beta 2$-specific $A b$ that was less efficient for precipitation than the previous lot. The new lot also precipitated a similar smaller percentage of ${ }^{125}$ I-epi-bound nAChRs when repeated with the $\alpha 4 \beta 2$ cell line (data not shown). The $\alpha 4$-specific polyclonal $\mathrm{Ab}$ precipitated the same percentage of the ${ }^{125} \mathrm{I}$-epi-bound $\mathrm{nAChRs}$ where the same lot of $\mathrm{Ab}$ was used. As with the $\alpha 4 \beta 2$ cell line, we observed a significant fold-increase in the percentage of precipitated ${ }^{125} \mathrm{I}$-epi binding for mAbs 270 and 299 with nicotine treatment. The fold-increase for both mAbs was twofold, which is smaller than that observed in the $\alpha 4 \beta 2$ cell line, consistent with the smaller fold-increase of upregulation (Fig. 6E). The percentage of the ${ }^{125} \mathrm{I}$-epi-bound nAChRs precipitated by mAbs 270 and 299 was higher than the $\alpha 4 \beta 2$ cell line. The higher percentage precipitated by the conformation-dependent mAbs may be caused by a higher efficiency of mature nAChR formation in the cortical neurons relative to the stable HEK cell line.

In a recent study, ${ }^{125} \mathrm{I}$-epi binding measurements were performed on brain tissue from nicotine- or saline-treated mice. Contrary to what we observed for the native nAChRs in cortical neurons, nicotine treatment did not result in increases in $\mathrm{mAb}$ 270 or 299 associations with native nAChRs (Marks et al., 2011). The differences between their findings and ours could result from a $2 \mathrm{~h}$ delay after nicotine treatment before processing the mice 
brains for epibatidine binding. In our cortical cultures, we found that $2 \mathrm{~h}$ after nicotine withdrawal, the fast component of upregulation had completely decayed and the fold-increase in ${ }^{125} \mathrm{I}$-epi binding changed from 2.9 to 1.7 (Fig. $2 B$ ). Thus, the measurements made on brain tissue would have missed the fast component and would have only measured the slow component of nicotine-induced upregulation. Consistent with this, ${ }^{125} \mathrm{I}$-epi binding in the cortical regions was increased only 1.5 -fold on average by the nicotine treatment when measured by immunoprecipitation, and 1.7-fold by autoradiography. In a different study (Turner et al., 2011), both the fast and slow components of nicotine-induced upregulation were observed when mice were killed without delay after a similar nicotine treatment and rapid membrane preparation. In this study, ${ }^{3} \mathrm{H}$-epi binding to the cortical regions was increased to significantly higher levels (2.7-fold) by nicotine treatment similar to the levels we measured before nicotine was withdrawn from the cortical cultures. Furthermore, they found that the binding had decayed $24 \mathrm{~h}$ after treatment to a level (1.6-fold) consistent with our measurements of the slow component and was maintained at that level for $3 \mathrm{~d}$. The results of these two studies would be consistent with our data on cortical cultures if a nicotine-induced $\alpha 4 \beta 2$ conformational change, as assayed by the conformation dependence of $\mathrm{mAb}$ precipitation of ${ }^{125}$ I-epi binding, is observed only during the rapid decay phase of the upregulation.

We, therefore, tested whether the nicotine-induced increases in $\mathrm{mAb}$ precipitations of $\mathrm{nAChRs}$ were only observed during the fast decay phase of the upregulation. For the cortical neurons, $5 \mathrm{~h}$ after nicotine withdrawal is a time when the fast component had completely disappeared and the slow component had not changed. Hence, $5 \mathrm{~h}$ after nicotine withdrawal we used the same sets of antibodies to assay changes in the percentage of ${ }^{125} \mathrm{I}$-epi counts precipitated. At this time after nicotine withdrawal, the nicotine-dependent increases in the percentage of precipitated ${ }^{125}$ I-epi-bound nAChRs had disappeared (Fig. 6 F), consistent with nicotine-dependent changes in epitope availability occurring as part of the fast component and not the slow component of upregulation. We also tested in $\alpha 4 \beta 2$ HEK cells whether conformational-dependent $\mathrm{mAb}$ precipitation of the ${ }^{125} \mathrm{I}$-epibound nAChRs changed with the decay of the fast component of the upregulation (Fig. 6E). At a time after nicotine withdrawal when the fast component had completely disappeared and the slow component had hardly changed $(3 \mathrm{~h})$, the nicotinedependent increases in the percentage of precipitated ${ }^{125} \mathrm{I}$-epibound nAChRs had disappeared (Fig. $6 E$ ). Thus, for the stably expressed $\alpha 4 \beta 2 \mathrm{nAChRs}$, as well as for the cortical neuron native $\alpha 4 \beta 2 \mathrm{nAChRs}$, the nicotine-dependent increases in mAb precipitation only occur during the fast phases of the nicotine-induced upregulation.

\section{Discussion}

While nicotine-induced upregulation of nAChRs is a well established phenomena linked to nicotine addiction, its role in nicotine addiction and the mechanisms responsible for upregulation are not established. Nonetheless, many different mechanisms have been proposed to explain how upregulation occurs. These include mechanisms that increase nAChR numbers such as a decrease in nAChR cell-surface turnover (Peng et al., 1994), increased nAChR trafficking to the surface (Darsow et al., 2005), increased nAChR subunit maturation and assembly in the ER (Harkness and Millar, 2002; Nashmi et al., 2003; Sallette et al., 2005), and block of nAChR subunit degradation in the ER (Ficklin et al., 2005; Rezvani et al., 2007). Additional mechanisms have been proposed to upregulate without increases in $\mathrm{nAChR}$ numbers such as a change in nAChR stoichiometry (Nelson et al., 2003; Moroni et al., 2006) and a nicotine-induced conformational change of $\mathrm{nAChRs}$ that alters the nAChR ligand binding state and function (Vallejo et al., 2005). In these studies, a single mechanism was assumed to be the predominant cause of upregulation, and the possibility of multiple mechanisms was not considered. Furthermore, all of the studies were performed on cells heterologously expressing nAChRs because of the difficulties involved in performing radiolabeled ligand-binding measurements and other assays on brain tissue. As a consequence, how these studies relate to the native brain nAChRs in neurons is unclear.

In this study, we characterized the nicotine-induced upregulation of $\alpha 4 \beta 2 \mathrm{nAChRs}$ in cultured cortical neurons by measuring the kinetics of upregulation of native nAChRs in real time and in living brain-derived neurons. During long exposures of the neurons to nicotine (17-24 h), ${ }^{125}$ I-epi binding increased at two rates with $\tau$ values of 1.1 and $12.5 \mathrm{~h}$. When nicotine was withdrawn, ${ }^{125}$ I-epi binding decayed at different rates, with $\tau$ values of $0.45 \mathrm{~h}$ and $12.3 \mathrm{~d}$. That the first process can occur independent of the second was demonstrated with shorter exposures of the cortical neurons to nicotine ( $\leq 4 \mathrm{~h}$ ) where only the fast decay occurred (Fig. 2C). During the initial process, nAChR numbers did not change and conformational-dependent binding of a set of nAChR-specific mAbs occurred (Fig. 6D). Conformationaldependent $\mathrm{mAb}$ binding remained only during the rapid decay and was gone subsequently. In addition, ${ }^{125} \mathrm{I}$-epi binding to cell-surface nAChRs rapidly dissipated (Fig. 3E), while the number of cellsurface nAChRs as assayed by immunoblot analysis remained essentially unchanged (Fig. 3D). Based on these results, we conclude that the initial component of upregulation results from nicotine-induced $\mathrm{nAChR}$ conformational changes. We assume that the conformational changes alter ${ }^{125}$ I-epi binding because they reflect nAChR transitions from a "resting" state with negligible ${ }^{125} \mathrm{I}$-epi binding to an "upregulated state" with high-affinity ${ }^{125}$ I-epi binding as previously proposed (Vallejo et al., 2005).

We also characterized mechanisms responsible for the second component of upregulation. During the onset of this process in HEK cells, we observed increased nAChR assembly (Fig. $4 A$ ) as previously observed (Sallette et al., 2005). A decreased rate of newly synthesized $\beta 2$ subunit degradation (Fig. $4 A-C$ ) accompanied nicotine-induced increases in $\mathrm{nAChR}$ assembly. Blocking proteasome-associated proteases increased subunit levels in cortical neurons and HEK cells (Fig. 5A,C, left). The proteasome inhibition also increased ${ }^{125}$ I-epi binding in HEK cells and cortical neurons consistent with increases associated with the second component of the upregulation (Fig. 5A, right, $C$, right). In addition, the decay rate of the second process in HEK cells $(\tau=44 \mathrm{~h}$; Fig. 2D) correlated with the rate of degradation of mature $\alpha 4 \beta 2$ nAChRs (mean $\tau=35$ h; Fig. 3D). Altogether, our results are consistent with a series of events starting with a mechanism protecting $\beta 2$ subunits from degradation by proteasomes (Rezvani et al., 2009). The resultant increased levels of $\beta 2$ subunits increases nAChR assembly in the ER and more intracellular and cellsurface nAChRs accumulate. The mechanisms causing the second component are, thus, distinct from those causing the first component of upregulation and result in significant changes in the number of upregulated $\alpha 4 \beta 2 \mathrm{nAChRs}$.

Aspects of upregulation, including its dose dependence and its kinetics, depended on the cell type (HEK cell vs cortical neurons) and subunit composition (Table 1). If the cortical neuron nAChR subunit composition is predominantly $\alpha 4$ and $\beta 2$ as indicated by our data (Fig. $1 B, C$ ), then other factors appear to cause the dif- 
ferences in $\alpha 4 \beta 2 \mathrm{nAChR}$ upregulation in the neurons from HEK cells. One can imagine that differences in post-translational modifications-phosphorylation (Bermudez and Moroni, 2006; Wecker et al., 2010), palmitoylation (Drisdel et al., 2004; Alexander et al., 2010), differences in interacting proteins (Jeanclos et al., 2001; Ficklin et al., 2005), or differences in the lipid environment (Fong and McNamee, 1986; Barrantes, 2010)_could cause changes in how upregulation occurs. One striking difference between native $\alpha 4 \beta 2 \mathrm{nAChRs}$ and the $\alpha 4 \beta 2 \mathrm{nAChRs}$ expressed in HEK cells is the faster onset of upregulation and threefold lower $\mathrm{EC}_{50}$ value for the native subunits. These findings suggest that nicotine can initiate upregulation of native $\alpha 4 \beta 2 \mathrm{nAChRs}$ in a matter of minutes at nicotine concentrations lower than previously thought. However, short-term exposure to nicotine, such as those achieved during one or two cigarettes, would only trigger the first component of upregulation, which rapidly reverses.

How do the results of this study using cultured cortical neurons relate to upregulation in vivo? While nAChRs in the cortical neurons are predominantly $\alpha 4 \beta 2 \mathrm{nAChRs,} \mathrm{the} \mathrm{situation} \mathrm{is} \mathrm{differ-}$ ent in the reward areas of the midbrain where dopamine neurons contain multiple nAChR subtypes, in particular $\alpha 6$-containing nAChRs (Gotti et al., 2005; Salminen et al., 2007), which are likely to complicate detailed studies of upregulation. Some studies using brain tissue have measured the decay rates of upregulated radioligand binding at different times after nicotine withdrawal. Decay rates varied somewhat depending on how nicotine was administrated or which brain region was studied (Marks et al., 1985; Collins et al., 1990, 1994; Hulihan-Giblin et al., 1990). Yet, upregulated binding decayed slowly, over days, similar to what we observed for the second component of upregulation. As discussed above, animals were killed $\geq 2 \mathrm{~h}$ after nicotine treatment ended, delaying the time between nicotine treatment and when radiolabeled agonist binding began. The delay allows nicotine metabolism to minimize the levels of nicotine and its metabolites present. However, a consequence of the delay is that the fast component of upregulation would have been missed due to its decay before binding measurements. Evidence of this is a recent study where radiolabeled agonist binding was assayed without delay and the fast decaying component of the upregulated binding was observed (Turner et al., 2011). Therefore, with the exception of one study, radiolabeled agonist binding measurements in brain tissue have only measured the slow decaying component of upregulation.

Nicotine-induced upregulation has been assumed to be caused by a single process. Instead, we find here that upregulation is composed of multiple processes occurring at very different rates with different underlying causes. Our findings that upregulation consists of a series of different processes stimulated by nicotine raise the possibility that upregulation has a more extensive role in nicotine addiction than previously thought. With very different kinetics, multiple upregulation components may contribute to different features of nicotine addictive behavior including sensitization, withdrawal, and tolerance, which occur at different times after nicotine exposure. This question would be ideally addressed if there existed a way to specifically block one component of upregulation without affecting other components. The specific blocker could then be applied to an in vivo preparation where the behavioral features of nicotine exposure can be measured for changes that correlate with the block. Another question raised by these findings is whether the fast initial component of upregulation is required for initiation of the slower second process or whether the second process is independent of the initial process. Indirect evidence that the two processes are linked is that the nicotine dose dependence of both processes together is described by a single binding isotherm with a Hill slope of 1.8 and an $\mathrm{EC}_{50}$ value of $92 \mathrm{~nm}$ for the cortical neuron nAChRs (Fig. 1A). If nicotine was acting through two independent processes, differences in the nicotine dose dependence would be expected, and we should have observed multiple components in the nicotine dose dependence or at least a Hill slope of $<1$. Other $\mathrm{nAChR}$ subtypes with different subunit compositions are upregulated by nicotine with similar steep nicotine dose dependences but with large differences in $\mathrm{EC}_{50}$ values for the nicotine dose dependence (Wang et al., 1998; Fenster et al., 1999; Tumkosit et al., 2006; Walsh et al., 2008). One of these subtypes, $\alpha 6 \beta 2 \mathrm{nAChRs}$ (Fig. $2 \mathrm{E}$ ), exhibits biphasic kinetics of the decay of ${ }^{125} \mathrm{I}$-epi binding similar to the kinetics of the cortical neuron (Fig. $2 B$ ) and stably expressed $\alpha 4 \beta 2 \mathrm{nAChRs}$ (Fig. $2 D$ ). The upregulation of $\alpha 6 \beta 2 \mathrm{nAChRs}$, like $\alpha 4 \beta 2 \mathrm{nAChRs}$, thus appears to consist of both components yet has a $>100$-fold difference in the $\mathrm{EC}_{50}$ value of the nicotine dependence of upregulation (Table 1). These findings suggest that the multiple processes underlying nicotine-induced upregulation are initiated by nicotine binding to the same binding sites on nAChRs.

\section{References}

Albuquerque EX, Pereira EF, Alkondon M, Rogers SW (2009) Mammalian nicotinic acetylcholine receptors: from structure to function. Physiol Rev 89:73-120.

Alexander JK, Govind AP, Drisdel RC, Blanton MP, Vallejo Y, Lam TT, Green WN (2010) Palmitoylation of nicotinic acetylcholine receptors. J Mol Neurosci 40:12-20.

Barrantes FJ (2010) Cholesterol effects on nicotinic acetylcholine receptor: cellular aspects. Subcell Biochem 51:467-487.

Benwell ME, Balfour DJ, Anderson JM (1988) Evidence that tobacco smoking increases the density of $(-)-\left[{ }^{3} \mathrm{H}\right]$ nicotine binding sites in human brain. J Neurochem 50:1243-1247.

Bermudez I, Moroni M (2006) Phosphorylation and function of alpha4beta2 receptor. J Mol Neurosci 30:97-98.

Breese CR, Marks MJ, Logel J, Adams CE, Sullivan B, Collins AC, Leonard S (1997) Effect of smoking history on $[3 \mathrm{H}]$ nicotine binding in human postmortem brain. J Pharmacol Exp Ther 282:7-13.

Buisson B, Bertrand D (2001) Chronic exposure to nicotine upregulates the human $\alpha 4 \beta 2$ nicotinic acetylcholine receptor function. J Neurosci 21:1819-1829.

Christianson JC, Green WN (2004) Regulation of nicotinic receptor expression by the ubiquitin-proteasome system. EMBO J 23:4156-4165.

Clarke PB, Schwartz RD, Paul SM, Pert CB, Pert A (1985) Nicotinic binding in rat brain: autoradiographic comparison of $[3 \mathrm{H}]$ acetylcholine, $[3 \mathrm{H}] \mathrm{ni}$ cotine, and [125I]- $\alpha$-bungarotoxin. J Neurosci 5:1307-1315.

Collins AC, Romm E, Wehner JM (1990) Dissociation of the apparent relationship between nicotine tolerance and up-regulation of nicotinic receptors. Brain Res Bull 25:373-379.

Collins AC, Luo Y, Selvaag S, Marks MJ (1994) Sensitivity to nicotine and brain nicotinic receptors are altered by chronic nicotine and mecamylamine infusion. J Pharmacol Exp Ther 271:125-133.

Conroy WG, Berg DK (1998) Nicotinic receptor subtypes in the developing chick brain: appearance of a species containing the alpha4, beta2, and alpha5 gene products. Mol Pharmacol 53:392-401.

Cui C, Booker TK, Allen RS, Grady SR, Whiteaker P, Marks MJ, Salminen O, Tritto T, Butt CM, Allen WR, Stitzel JA, McIntosh JM, Boulter J, Collins AC, Heinemann SF (2003) The $\beta 3$ nicotinic receptor subunit: a component of $\alpha$-conotoxin MII-binding nicotinic acetylcholine receptors that modulate dopamine release and related behaviors. J Neurosci 23:11045-11053.

Darsow T, Booker TK, Piña-Crespo JC, Heinemann SF (2005) Exocytic trafficking is required for nicotine-induced up-regulation of alpha 4 beta 2 nicotinic acetylcholine receptors. J Biol Chem 280:18311-18320.

De Biasi M, Dani JA (2011) Reward, addiction, withdrawal to nicotine. Annu Rev Neurosci 34:105-130.

Drisdel RC, Green WN (2000) Neuronal alpha-bungarotoxin receptors are $\alpha 7$ subunit homomers. J Neurosci 20:133-139.

Drisdel RC, Manzana E, Green WN (2004) The role of palmitoylation in functional expression of nicotinic $\alpha 7$ receptors. J Neurosci 24:10502-10510. 
Fenster CP, Whitworth TL, Sheffield EB, Quick MW, Lester RA (1999) Upregulation of surface $\alpha 4 \beta 2$ nicotinic receptors is initiated by receptor desensitization after chronic exposure to nicotine. J Neurosci 19:4804-4814.

Ficklin MB, Zhao S, Feng G (2005) Ubiquilin-1 regulates nicotine-induced up-regulation of neuronal nicotinic acetylcholine receptors. J Biol Chem 280:34088-34095.

Fong TM, McNamee MG (1986) Correlation between acetylcholine receptor function and structural properties of membranes. Biochemistry $25: 830-840$.

Gotti C, Moretti M, Clementi F, Riganti L, McIntosh JM, Collins AC, Marks MJ, Whiteaker P (2005) Expression of nigrostriatal alpha 6-containing nicotinic acetylcholine receptors is selectively reduced, but not eliminated, by beta 3 subunit gene deletion. Mol Pharmacol 67:2007-2015.

Govind AP, Vezina P, Green WN (2009) Nicotine-induced upregulation of nicotinic receptors: underlying mechanisms and relevance to nicotine addiction. Biochem Pharmacol 78:756-765.

Grailhe R, de Carvalho LP, Paas Y, Le Poupon C, Soudant M, Bregestovski P, Changeux JP, Corringer PJ (2004) Distinct subcellular targeting of fluorescent nicotinic alpha 3 beta 4 and serotoninergic 5-HT3A receptors in hippocampal neurons. Eur J Neurosci 19:855-862.

Green WN, Claudio T (1993) Acetylcholine receptor assembly: subunit folding and oligomerization occur sequentially. Cell 74:57-69.

Härfstrand A, Adem A, Fuxe K, Agnati L, Andersson K, Nordberg A (1988) Distribution of nicotinic cholinergic receptors in the rat tel- and diencephalon: a quantitative receptor autoradiographical study using [3H]acetylcholine, [alpha-125I]bungarotoxin and [3H]nicotine. Acta Physiol Scand 132:1-14.

Harkness PC, Millar NS (2002) Changes in conformation and subcellular distribution of $\alpha 4 \beta 2$ nicotinic acetylcholine receptors revealed by chronic nicotine treatment and expression of subunit chimeras. J Neurosci 22:10172-10181.

Hsu YN, Amin J, Weiss DS, Wecker L (1996) Sustained nicotine exposure differentially affects alpha 3 beta 2 and alpha 4 beta 2 neuronal nicotinic receptors expressed in Xenopus oocytes. J Neurochem 66:667-675.

Hulihan-Giblin BA, Lumpkin MD, Kellar KJ (1990) Effects of chronic administration of nicotine on prolactin release in the rat: inactivation of prolactin response by repeated injections of nicotine. J Pharmacol Exp Ther 252:21-25.

Jeanclos EM, Lin L, Treuil MW, Rao J, DeCoster MA, Anand R (2001) The chaperone protein 14-3-3eta interacts with the nicotinic acetylcholine receptor alpha 4 subunit. Evidence for a dynamic role in subunit stabilization. J Biol Chem 276:28281-28290.

Karlin A, Akabas MH (1995) Towards a structural basis for the function of nicotinic acetylcholine receptors and their cousins. Neuron 15:1231-1244.

Klink R, de Kerchove d'Exaerde A, Zoli M, Changeux JP (2001) Molecular and physiological diversity of nicotinic acetylcholine receptors in the midbrain dopaminergic nuclei. J Neurosci 21:1452-1463.

Marks MJ, Burch JB, Collins AC (1983) Effects of chronic nicotine infusion on tolerance development and nicotinic receptors. J Pharmacol Exp Ther 226:817-825.

Marks MJ, Stitzel JA, Collins AC (1985) Time course study of the effects of chronic nicotine infusion on drug response and brain receptors. J Pharmacol Exp Ther 235:619-628.

Marks MJ, McClure-Begley TD, Whiteaker P, Salminen O, Brown RW, Cooper J, Collins AC, Lindstrom JM (2011) Increased nicotinic acetylcholine receptor protein underlies chronic nicotine-induced up-regulation of nicotinic agonist binding sites in mouse brain. J Pharmacol Exp Ther 337:187-200.

McGehee DS, Role LW (1995) Physiological diversity of nicotinic acetylcholine receptors expressed by vertebrate neurons. Annu Rev Physiol 57:521-546.

Merlie JP, Lindstrom J (1983) Assembly in vivo of mouse muscle acetylcholine receptor: identification of an alpha subunit species that may be an assembly intermediate. Cell 34:747-757.

Moroni M, Zwart R, Sher E, Cassels BK, Bermudez I (2006) alpha4beta2 nicotinic receptors with high and low acetylcholine sensitivity: pharmacology, stoichiometry, and sensitivity to long-term exposure to nicotine. Mol Pharmacol 70:755-768.

Nashmi R, Dickinson ME, McKinney S, Jareb M, Labarca C, Fraser SE, Lester HA (2003) Assembly of $\alpha 4 \beta 2$ nicotinic acetylcholine receptors assessed with functional fluorescently labeled subunits: effects of localization, traf- ficking, and nicotine-induced upregulation in clonal mammalian cells and in cultured midbrain neurons. J Neurosci 23:11554-11567.

Nelson ME, Kuryatov A, Choi CH, Zhou Y, Lindstrom J (2003) Alternate stoichiometries of alpha4beta2 nicotinic acetylcholine receptors. Mol Pharmacol 63:332-341.

Parker SL, Fu Y, McAllen K, Luo J, McIntosh JM, Lindstrom JM, Sharp BM (2004) Up-regulation of brain nicotinic acetylcholine receptors in the rat during long-term self-administration of nicotine: disproportionate increase of the alpha6 subunit. Mol Pharmacol 65:611-622.

Pauly JR, Stitzel JA, Marks MJ, Collins AC (1989) An autoradiographic analysis of cholinergic receptors in mouse brain. Brain Res Bull 22:453-459.

Peng X, Gerzanich V, Anand R, Whiting PJ, Lindstrom J (1994) Nicotineinduced increase in neuronal nicotinic receptors results from a decrease in the rate of receptor turnover. Mol Pharmacol 46:523-530.

Perry DC, Dávila-García MI, Stockmeier CA, Kellar KJ (1999) Increased nicotinic receptors in brains from smokers: membrane binding and autoradiography studies. J Pharmacol Exp Ther 289:1545-1552.

Rezvani K, Teng Y, Shim D, De Biasi M (2007) Nicotine regulates multiple synaptic proteins by inhibiting proteasomal activity. J Neurosci 27:10508-10519.

Rezvani K, Teng Y, Pan Y, Dani JA, Lindstrom J, García Gras EA, McIntosh JM, De Biasi M (2009) UBXD4, a UBX-containing protein, regulates the cell surface number and stability of $\alpha 3$-containing nicotinic acetylcholine receptors. J Neurosci 29:6883-6896.

Rogers SW, Gregori NZ, Carlson N, Gahring LC, Noble M (2001) Neuronal nicotinic acetylcholine receptor expression by $\mathrm{O} 2 \mathrm{~A} /$ oligodendrocyte progenitor cells. Glia 33:306-313.

Sallette J, Pons S, Devillers-Thiery A, Soudant M, Prado de Carvalho L, Changeux JP, Corringer PJ (2005) Nicotine upregulates its own receptors through enhanced intracellular maturation. Neuron 46:595-607.

Salminen O, Drapeau JA, McIntosh JM, Collins AC, Marks MJ, Grady SR (2007) Pharmacology of alpha-conotoxin MII-sensitive subtypes of nicotinic acetylcholine receptors isolated by breeding of null mutant mice. Mol Pharmacol 71:1563-1571.

Sargent PB (1993) The diversity of neuronal nicotinic acetylcholine receptors. Annu Rev Neurosci 16:403-443.

Schwartz RD, Kellar KJ (1983) Nicotinic cholinergic receptor binding sites in the brain: regulation in vivo. Science 220:214-216.

Sheffield EB, Quick MW, Lester RA (2000) Nicotinic acetylcholine receptor subunit mRNA expression and channel function in medial habenula neurons. Neuropharmacology 39:2591-2603.

Tumkosit P, Kuryatov A, Luo J, Lindstrom J (2006) Beta3 subunits promote expression and nicotine-induced up-regulation of human nicotinic alpha6 $6^{*}$ nicotinic acetylcholine receptors expressed in transfected cell lines. Mol Pharmacol 70:1358-1368.

Turner JR, Castellano LM, Blendy JA (2011) Parallel anxiolytic-like effects and upregulation of neuronal nicotinic acetylcholine receptors following chronic nicotine and varenicline. Nicotine Tob Res 13:41-46.

Vallejo YF, Buisson B, Bertrand D, Green WN (2005) Chronic nicotine exposure upregulates nicotinic receptors by a novel mechanism. J Neurosci 25:5563-5572.

Vezina P, McGehee DS, Green WN (2007) Exposure to nicotine and sensitization of nicotine-induced behaviors. Prog Neuropsychopharmacol Biol Psychiatry 31:1625-1638.

Walsh H, Govind AP, Mastro R, Hoda JC, Bertrand D, Vallejo Y, Green WN (2008) Up-regulation of nicotinic receptors by nicotine varies with receptor subtype. J Biol Chem 283:6022-6032.

Wang F, Nelson ME, Kuryatov A, Olale F, Cooper J, Keyser K, Lindstrom J (1998) Chronic nicotine treatment up-regulates human alpha3 beta2 but not alpha3 beta 4 acetylcholine receptors stably transfected in human embryonic kidney cells. J Biol Chem 273:28721-28732.

Wecker L, Pollock VV, Pacheco MA, Pastoor T (2010) Nicotine-induced up regulation of alpha4beta2 neuronal nicotinic receptors is mediated by the protein kinase C-dependent phosphorylation of alpha4 subunits. Neuroscience 171:12-22.

Whiting PJ, Lindstrom JM (1988) Characterization of bovine and human neuronal nicotinic acetylcholine receptors using monoclonal antibodies. J Neurosci 8:3395-3404.

Xu W, Orr-Urtreger A, Nigro F, Gelber S, Sutcliffe CB, Armstrong D, Patrick JW, Role LW, Beaudet AL, De Biasi M (1999) Multiorgan autonomic dysfunction in mice lacking the $\beta 2$ and the $\beta 4$ subunits of neuronal nicotinic acetylcholine receptors. J Neurosci 19:9298-9305. 